mLE: LOW MASS SN IA AND THE LATE LIGHT CURVE

OSTI

AUTHOR(5): Stirling A. Colgate, T-6

Chris L. Fryer, Univ of Arizona

Kevin P. Hand, Dartmouth College

SUBMTTEDTO: NATO ADVANCED STUDY INSTITUTE ON

THERMONUCLEAR SUPERNOVAE

June 19 - 30, 1995, Barcelona, Spain
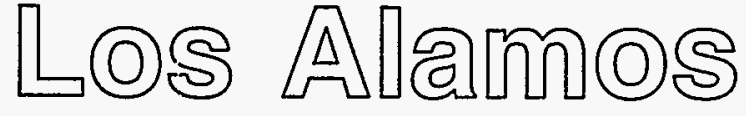

FORM NO. 836 R4

ST. NO. $26295 / 81$

\section{Los Alamos National Laboratory Los Alamos, New Mexico 87545}

\section{MASTER}

DISTRIDUTION OF THIS DCOMMENT IS UNLMITED 


\title{
LOW MASS SN IA AND THE LATE LIGHT CURVE
}

\author{
STIRLING A. COLGATE \\ Theoretical Division, T-6, MS B275 \\ Los Alamos National Laboratory \\ Los Alamos, NM 87545 \\ CHRIS L. FRYER \\ Steward Observatory \\ University of Arizona \\ Tucson, $A Z 85721$ \\ AND \\ KEVIN P. HAND \\ Dartmouth College \\ HB 1985 \\ Hanover, New Hampshire 03755
}

\begin{abstract}
.
The late bolometric light curves of type Ia supernovae, when measured accurately over several years, show an exponential decay with a 56d halflife over a drop in luminosity of 8 magnitudes ( 10 half-lives). The late-time light curve is thought to be governed by the decay of $\mathrm{Co}^{56}$, whose $77 \mathrm{~d}$ half-life must then be modified to account for the observed decay time. Two mechanisms, both relying upon the positron fraction of the $\mathrm{Co}^{56} \mathrm{de}$ cay, have been proposed to explain this modification. One explanation requires a large amount of emission at infra-red wavelengths where it would not be detected(Axelrod, 1980a,b). The other explanation has proposed a progressive transparency or leakage of the high energy positrons (Colgate, Petschek and Kriese, 1980). For the positrons to leak out of the expanding nebula at the required rate necessary to produce the modified $56 \mathrm{~d}$ exponential, the mass of the ejecta from a one foe $\left(10^{51} \mathrm{erg}\right.$ in kinetic energy) explosion must be small, $M_{e j e c}=0.4 M_{\odot}$ with $M_{e j e c} \propto K E^{0.5}$. Thus, in this leakage explanation, any reasonable estimate of the total energy of the explosion requires that the ejected mass be very much less than the Chandrasekhar mass of $1.4 M_{\odot}$. This is very difficult to explain with the "canonical" Chandrasekhar-mass thermonuclear explosion that
\end{abstract}

\section{DISCLAIMER}

This report was prepared as an account of work sponsored by an agency of the United States Government. Neither the United States Government nor any agency thereof, nor any of their employees, makes any warranty, express or implied, or assumes any legal liability or responsibility for the accuracy, completeness, or usefulness of any information, apparatus, product, or process disclosed, or represents that its use would not infringe privately owned rights. Reference herein to any specific commercial product, process, or service by trade name, trademark, manufacturer, or otherwise does not necessarily constitute or imply its endorsement, recommendation, or favoring by the United States Government or any agency thereof. The views and opinions of authors expressed herein do not necessarily state or reflect those of the United States Government or any agency thereof. 
disintegrates the original white dwarf star. This result leads us to pursue alternate mechanisms of type Ia supernovae. These mechanisms include sub-Chandrasekhar thermonuclear explosions and the accretion induced collapse of Chandrasekhar mass white dwarfs. We will summarize the advantages and disadvantages of both mechanisms with considerable detail spent on our new accretion induced collapse simulations. These mechanisms lead to lower $\mathrm{Ni}^{56}$ production and hence result in type Ia supernovae with luminosities decreased down to $\sim 50 \%$ that predicted by the "standard" model.

\section{Introduction}

\subsection{HISTORICAL PERSPECTIVE}

Since humans first started observing the heavens, the light emission from supernovae has attracted their attention. The same properties that first instigated this interest (a time history comparable to the human attention span and a radiation spectrum matching the sensitivity of the human eye) formed the basis of the difficulties behind solving the physical mechanisms for these objects. The energies required to induce an explosion $(\sim \mathrm{MeV}$ per nucleon) and the proposed mechanisms for the outbursts invoking either a thermonuclear explosion (Burbidge et al., 1957) or a collapse into a neutron star (Zwicky, 1938) seemed to defy the soft $(\sim \mathrm{eV})$ photons observed. Assuming stellar opacities and stellar structure analysis (Minkowski, 1964) required extraordinary energies to explain the two to three week rise and fall times for type Ias. Dedicated observations of several close, bright supernovae (particularly 1937E, Baade and Zwicky, 1938) showed that the late time light curve decayed in half a dozen examples with the extraordinary time constant of $\sim 56$ days. An exponential over 8 magnitudes was clearly a strong indication of a simple physical process. The race was then on in the late 30's to find this simple physical process which would then provide the physical mechanism(s) of these stellar explosions. Unfortunately a simple explanation was not forthcoming, despite such enthusiastic suggestions as the decay of radioactive californium with its $\sim 54 \mathrm{~d}$ half life (Baade et al., 1956). This, however, would have swamped the Galaxy in transuranic elements and fission products. When these fires had burned out, the puzzle passed to the numerical modelers. Finding a reasonable explanation for the mechanism of the supernova explosion as neutrino transport of the neutron star binding energy (Colgate \& White, 1966)) was relatively straightforward compared to the seemingly impossible task of extracting from this mechanism any emission of light. The problem, recognized in $\mathrm{C \& W}$, was, and 
still is, that the internal energy (from neutrino deposition, thermonuclear and the explosion shock), although $\sim 100$ times the emitted luminosity, is nevertheless converted by adiabatic expansion into kinetic energy, leaving less than $10^{-4} \%$ of the initial energy (assuming a compact $R \sim 10^{9} \mathrm{~cm}$ stellar progenitor) by the time the optical depth becomes low enough for the photons to escape and "light" the supernova. Only for larger progenitors ( $R \sim 10^{13}$ red giant stars) was there any hope for powering the light curve by the initial explosion energy. The problem changed from finding a mechanism to explain the 56d light curve to finding a way for one of the existing mechanisms to produce any light at all. After all, a supernova without light is about as interesting as the sound of one hand clapping.

What was needed was a late source of energy to heat the expanding debris after most of the expansion, but before the diffusive release of the photons took place. Having initially failed to explain the 56d light curve, radioactive decays were now called upon (C\&W) to solve the more critical problem of finding a power source for the maximum and total luminosity.

Without the foreknowledge of the unique properties of the maximum bound-mass alpha-particle nucleus, $\mathrm{Ni}^{56}$, a statistical ensemble of radioactive decays or excited nuclear states of all abundant nuclei ejected in the explosion was assumed. By definition, this must include allowed as well as non-allowed transitions. The resulting distribution of decays led to a powerlaw in energy decay rate, $\propto t^{-1.4}$ starting at $t_{0} \sim 0.1 \mathrm{~s}$ in time. Thus, by the time of light maximum, the initial, integral, radioactive energy would have decayed by $\left(t_{0} / t_{\text {max }}\right)^{-0.4} \sim 10^{-3}$. This was even worse than the adiabatic expansion loss, because the initial radioactive energy was far less than the $\mathrm{MeV}$ per nucleon value obtained from the initial internal energies.

Another possible explaination was that at the time of maximum light, the expanding matter collided with a nebula of $\sim 0.01 M_{\odot}$ extending radially outwards from $100 \mathrm{AU}$. This seemed equally unlikely at that time. Thus the neutrino model was published with only the one hand clapping.

The reason for mentioning these failed light sources is to recognize the unique properties of the nucleus $\mathrm{Ni}^{56}$ for powering the supermova light curve. The daughter nucleus, $\mathrm{Co}^{56}$, is a high energy forbidden decay, $4.6 \mathrm{Mev}$, with a very long half life $(\sim 77 d)$. This is longer by $\sim 10^{7}$ times the typical allowed transition. The isotope is made abundantly as the end point of alpha particle nuclear synthesis, and results in the common abundant element iron. Hence, the decay of $\mathrm{Ni}^{56}$ avoids the nucleosynthetic waste problems afflicting the previous model involving the decay of radioactive californium. This recognition at a conference on nuclear synthesis at Boulder Co. (Colgate and McKee, 1969) led to the "second" hand for Type Ia supernova mechanisms. Although the energy source was reasonable, one sore thumb remained, namely, that the $77 \mathrm{~d}$ half-life was not the $56 \mathrm{~d}$ half-life observed. 
Attention shifted from this problem to the spectra and the light curve near peak luminosity. There was good agreement on the spectra between thermonuclear models using a $1.4 M_{\odot}$ mixture of silicon burning elements and the many detailed spectral observations and the light curve shape within a magnitude down from maximum. This gave strong support to the ideas that $\mathrm{Ni}^{56}-\mathrm{Co}^{56}$ powered the luminosity and for the Chandrasekhar-mass thermonuclear explosion model of type las. Thus the solution of one problem, the source of the optical energy, seemed solved. However, the original problem of the $56 \mathrm{~d}$ decay remained. In addition the decay of the luminosity after peak of the $1.4 M_{\text {odot }}$ models was somewhat slower than the observed "fast" SN1As.

The problem of the late-time light curve, particularly as observed by Kirshner and Oke (1975) and Baade and Zwicky, (1938) has been discussed in some depth by Colgate and Petschek (1980), Axelrod,(1980a) and Weaver, Axelrod and Woosley, (1980) and Colgate, Petschek and Kriese, (1980), (herafter, CPK) Axelrod (1980b), Colgate and Petschek, (1982) and Colgate (1991), Kirshner, (1990), Dogget and Branch (1985), and Wheeler, Harkness, and Khokhlov (1995). Two explainations have been given for the modification of the $77 \mathrm{~d}$ radioactive decay half life to the faster $56 \mathrm{~d}$ half-life of the late time luminosity (after about $100 \mathrm{~d}$ ). Both require the non-optical radiation loss of radioactive decay energy from the luminous nebula. The first (CPK) proposed the late time escape from the nebula of a progressively increasing fraction of the positrons from $\mathrm{Co}^{56}$. In the second case Axelrod proposed that all the energy of the positrons was deposited in the luminous nebula, but that a radiation "infrared catastrophy" occured that emitted the late time energy as infrared emission from low lying forbidden transitions of FeII.

\subsection{PLAN OF PAPER}

Section II of this paper is concerned primarily with the positron escape explanation of the $56 \mathrm{~d}$ decay period. The dominant physical effects are presented and discussed. We will also argue against the "infrared catastrophe" explanation and provide additional support for the positron escape model. Section III will discuss the results from our one-zone simulations leading to the requirement of low mass ejection and hence its implication for an alternate explanation for the type Ia explosion mechanism. The problem of spectra is not treated in depth in this paper, but the next section concludes with an argument why one expects the spectra to be a weak discriminant of ejected mass. Section IV will conclude this work by discussing possible supernova mechanisms for ejecting low mass with detailed simulations of the accretion induced collapse scenario. 


\section{The Physics of the Supernova Light Curves}

A "strong" explosion shockwave deposits internal energy and kinetic energy equally in the envelope of the pre-SN star. Because the radiation is initially trapped, the ejecta expands adiabatically, converting internal energy to kinetic energy. Throughout the expansion, $a T^{4} \gg n k T$ and thus the total internal energy can be approximated by $E_{T} \propto 1 / R \propto 1 / t$ (when the velocity of the expansion approaches a constant and $\left.\gamma_{g a s}=4 / 3\right)$.

We have built a single zone model using the calculating and graphics routine, Kaladagraph (reg.) so that many models and approximations may be explored. In particular, a single zone model allows us to explore the latetime light curve where the envelope is optically thin without the extensive calculations associated with diffusion. To compare with the many multizone calculations, we use the W7 thermonuclear model of Nomoto (1984) $\left(M_{0}=1.4 M \odot, R_{0}=2 \times 10^{8} \mathrm{~cm}\right.$, and $E_{K}$, the kinetic plus internal energy or 1.3 foe, $\left(\times 10^{51}\right.$ ergs)). After the initial shock where the kinetic and internal energy are divided equally, adiabatic expansion returns all but a small fraction of the internal energy to kinetic energy. This kinetic energy results in the free expansion of $M_{e j}$ at an asymtotically limiting velocity, $v_{o}$, at an outer boundary, $\mathrm{R}$, where $R=v_{o} t$. For a uniform density sphere $E_{K}=(3 / 5) M_{e j}\left(v_{o}^{2} / 2\right)$. In the real case of an exploding star, the outer bounday expands significantly faster than this because of a combination of a rarefaction wave during expansion as well as the speed-up of the initial shock in the density gradient of the envelope ( $C \& W)$. Consequently the inner core has given up significant energy to the outer, faster moving matter, leading to a density distribution of the outer layers of $\rho=\rho_{(t)}(r / R)^{-7}$ where $I$ is the Lagrange radius within the nebula $(C \& W)$ and $\rho_{(t)}$ is the time dependent density of the inner uniform mass fraction. We estimate this energy fraction as $20 \%$ so that the effective expansion is $10 \%$ slower, or $E_{K}=(0.8) M_{e j}\left(v_{o}^{2} / 2\right)$.

Clearly the single zone model will not calculate diffusion and thus only give an approximation to the peak luminosity. However, as pointed out by Arnett (1982), diffusion is no longer important after the peak and the light curve is determined by the radioactive energy deposition. This is the region of our primary interest.

Initially we demonstrate an adiabatic expansion where the only loss is PdV work. Figure 1 shows the total internal energy on a log-log plot versus time. Since the shock is freely expanding $\left(R=v_{o} t\right), E_{\text {int }} \propto 1 / R \propto 1 / t$ which verifies our calculation of the adiabatic expansion. The initial value of $E_{\text {int }}$ corresponds to the shock deposited energy $\left(\frac{1}{2} \times 1.3\right.$ foe initially deposited in uniform density at $R_{0}=2 \times 10^{8} \mathrm{~cm}$ and expanded at $v_{0}$ for 1 day). The top curve shows the internal energy with the addition of the 
energy from the radioactive decay of $\mathrm{Ni}^{56}$ added to the adiabatic expansion loss. The very large, $\sim 10^{4}$, difference between these curves emphasizes the extreme importance of the radioactive energy source for the supernova luminosity. Two intermediate curves show the effect of including the two energy losses: (1) the escape of radioactive particles from the nebula and (2) the diffusion of initially trapped radiant energy. We consider the diffusive loss first, because it is here that we expect the greatest departure from reality for a single zone model.

\subsection{DIFFUSION AND LIGHT MAXIMUM}

The photons will diffuse out of the nebula and decrease the internal energy. A "diffusion wave" of thickness $\Delta R=\left(D_{\text {diff }} t\right)^{1 / 2}$ penetrates into the surface where $D_{\text {diff }}$ is the diffusion coefficient. Since $D_{\text {diff }} \propto \rho^{-1} \propto t^{3}$, $\Delta R \propto t^{2}$ and thus the diffusion wave leads to a rapid uncovering of the initially trapped internal energy as the nebula expands. However, as we have already noted, the adiabatic expansion reduces the initial internal energy before it can diffuse outwards, but now the internal energy is augmented by $\dot{W}$, the instantaneous radioactive energy deposited in the nebula. The luminosity can then be given as

$$
L=E_{T} / t_{\text {diff }}
$$

where $t_{\text {diff }}=R \tau /(\pi c / 3)$, the characteristic diffusion time for a uniform expanding sphere. Here $\tau=R \rho \kappa$ and $\kappa$ is the radiation opacity. Then the difference equation with time $=n$ and the time step $\Delta t$, for the energy becomes

$$
E_{T(n)}=E_{T(n-1)}-\left(E_{T(n-1)} / t\right) \Delta t+\dot{W}_{\text {dep }} \Delta t-\left(E_{T(n-1)} / t_{d i f f}\right) \Delta t .
$$

Here the second term on the right hand side is the PdV work leading to the adiabatic expansion for $\gamma=4 / 3$, followed by the radioactive energy deposition, and then the diffusive loss. Eq. (2) is then integrated until $\dot{W}_{\text {dep }} \geq L$ from eq. (1). Thereafter $L=\dot{W}_{\text {dep }}$.

Although eqs. (1 and 2) are a simplistic approximation to the diffusion equations especially at early times, after the peak luminosity the diffusion timescale decreases rapidly. At the late times which are the primary issue of this paper, the diffusion timescale becomes so short that the energy is released as soon as it is added to the system and the luminosity is indeed very close to the energy deposition rate. We can use these simple equations to more easily interpret and intuit the observations. However, we can also use these simple equations at early times to develop our intuition on specific aspects of the light curve physics such as the effects of expansion on the opacity. 
A common misconception in type Ia light curve calculations is that the width of the light curve depends sensitively upon the mass of the ejecta $\left(M_{\text {eject }}\right)$. The expansion opacity from line scattering in an expanding medium to first approximation cancels the effect of $M_{\text {eject }}$ on the time to maximum light for the particular range of $M_{\text {eject }} \lesssim 2 M_{\odot}$ where the lower mass limit is not known. In the next section, we will show why the difference in times to maximum luminosity for the range of $M_{\text {eject }}$ for the various type Ia mechanisms is small and within the uncertainty of the observations.

\subsection{EXPANSION OPACITY}

The compensating effect of the expansion opacity for the rise time of low mass ejection was discussed in Colgate (1989). Karp et al. (1977) showed how the opacity of an expanding media was different from static atmospheres due to the monotonic redshift of every photon. Because there is a gradient in the expansion velocity, as the photon travels through the expanding nebula, its apparent wavelength in the rest frame at different radii will vary. This continuous range of redshifts allows for the possibility of a photon to scatter from a sequence of atomic states as it traverses the medium before it is then Compton scattered by a background of free electrons. This "expansion opacity" can increase the opacity above the Compton opacity by orders of magnitude in a limited frequency range. However, this requires that the expansion velocity gradient is sufficiently large over a Compton mean free path to cause the photon to be redshifted through a number of strong atomic lines. In other words, the fractional energy gap between states must be less than the redshift of the photons in the Compton mean free path. This fractional redshift is measured as $1 / \mathrm{s}$ where $\mathrm{s}$, the expansion parameter, is

$$
s=\kappa_{c} \rho c t \propto \kappa_{c} M_{\text {eject }} c t^{-2} /\left(4 \pi v_{o}^{3} / 3\right) .
$$

The calculation of Karp et al. used a set $N \sim 2.6 \times 10^{5}$ lines within a temperature range $6000 \mathrm{~K}<T<30,000 \mathrm{~K}$. The opacity enhancement factor, $\Omega$ above the Compton opacity depends upon (1) ionization for free electrons, (2) the thermal production of excited states, and (3) the number of gaps in this "forest" of lines. Hence enhancement occurs only for $1 / s>N^{1 / 2}$, or $s<1000$. For very small mass ejecta or at very late times, the low density will give a low value for $s$ (a large change in velocity over a Compton mean free path). However, if the density gets too low, the weak lines will be insufficient to cover gaps in the Rosseland mean and the enhancement factor will no longer increase. We can express the opacity as:

$$
\kappa \sim \kappa_{T} \Omega \text {. }
$$


Here $\kappa_{T}$ is a temperature dependent function that convolves the density of states and line strengths averaged over composition. More detailed calculations have been performed of the expansion opacity particularly by Harkness (1991) and Höflich, Khokhlov, and Wheeler (1995), and we use an analytical approximation for $\kappa_{T}$ from Höflich (1990), shown in Fig. 2, where the enhancement factor is:

$$
\Omega=(1+700 / s)
$$

and with $\Omega \leq 10$.

This gives similar results to Karp et al. (1977), but the greater number of lines used in the later calculations result in a smoother function. For $s$ small then $\Omega \propto 1 / s$. As a consequence the optical depth becomes

$$
\tau=\kappa \rho R \propto \kappa T v_{0}
$$

which is indepedent of $t$. We can thus estimate the maximum luminosity to occur after a characteristic diffusion time. The diffusion time $t_{\text {diff }} \propto$ $R \tau \propto v_{o} \tau \propto v_{o}^{2} \kappa_{T}$ and hence the condition for light maximum depends on $\kappa_{T} v_{o}^{2}$. To the extent that $v_{o}^{2}$ is independent of the mass, the time to peak luminosity depends primarily upon $\kappa_{T}$. Since $\kappa_{T}$ depends primarily upon temperature with a sharp cut-off below $\sim 6000 K$, the peak luminosity will occur when the temperature falls below $6000 \mathrm{~K}$. The temperature, in turn, depends upon the volume and the deposited radioactive energy. With the volume fixed at a given time and for a fixed $v_{0}$, the temperature will scale as $M_{N i}^{1 / 4}$, a weak dependence upon ejected mass. As we shall demonstrate later, we have compared a single zone model calculation of Nomoto's W7 progenitor and the calculations of Harkness et al. to a single zone model of a low mass progenitor $\left(M_{\text {eject }}=0.4 M_{\odot}=28 \% M_{W 7}\right)$. The resulting rise times to peak are quite similar (15 and 13.5 days respectively).

Before we begin a full discussion of these calculations, we must first consider the loss of energy due to the escape of gamma-rays and positrons from the expanding nebula.

\subsection{THE DEPOSITION OF GAMMA-RAYS AND POSITRONS}

Gamma-rays and positrons from the decay of $\mathrm{Ni}^{56}$ deposit their energy in the expanding nebula as a function of their respective absorption coefficients. The first calculation of this deposition by CPK used the Los Alamos Monte Carlo code for gamma-ray transport and it accounted in detail for the effects of Compton scattering, build-up factors and multiple energy gamma-rays. They found that a relatively simple analytic expression surprisingly reproduced the very complicated effects of the Monte Carlo calculation independent of whether the source function was distributed within 
the inner half or outer half radius of a homogeneous sphere. The reason for this simplicity is that when the ejecta is thick, nearly all the gamma-rays are absorbed. There is then an analytic expression for the deposition. Similarly, when the ejecta is thin, the absorbed fraction is a simple analytic expression $\propto \tau$. The deposition fraction for $\tau>0.25$ given in CPK is

$$
D_{\text {dep }}=G(1+2 G(1-G)(1-0.75 G))
$$

where $G=\frac{\tau}{1.6+\tau}$ and the deposition fraction for $\tau \leq 0.25$

$$
D_{\text {dep }}=0.64 \tau \text {. }
$$

Here $\tau$ is the thickness of the nebula to either gamma rays or positrons. For gamma rays we use an "effective opacity" including the "build-up factor" from the Monte Carlo calculations of $\kappa_{\gamma}=35.5 \mathrm{~cm}^{2} \mathrm{~g}^{-1}$ from CPK for ejecta that is primarily silicon burning products, $Y_{e}=0.5$. This agrees within $3 \%$ with the independent multi-zone calculations of W7 by Swartz et. al. (1995). For larger mass models, presumably type IIs with a major fraction of hydrogen we use $\kappa_{\gamma}=28.8 \mathrm{~cm}^{2} \mathrm{~g}^{-1}$. Since the derivation of this approximation for the deposition fraction, many calculations of the deposition of gamma-rays have been made using migrated versions of the original Los Alamos Monte Carlo code. Swartz, Sutherland \& Harkness (1995) derived an alternative analytic approximation which showed good agreement with their Monte Carlo calculations. However, they made no comparisons with any earlier work. In Fig. 3 we give this comparison which indicates negligible difference with the simple function given by eq. (7) for the critical region of the transition from very thick to thin. The thin approximation of Swartz et al (1995), their figure 9, also agrees with eq. (8) to within $10 \%$ at late times.

\subsection{THE MODIFIED EXPONENTIAL BY DEPOSITION}

The deposition fraction will modify the decay of the luminosity and therefore, from eqs. (1) and (2), the apparent decay rate of the radioactive excitation function. The increasing fraction of escaping or lost energy appears as a more rapid exponential radioactive decay rate. Recall, we are seeking a mechanism to modify the $77 \mathrm{~d}$ decay rate of $\mathrm{Co}^{56}$ to match the $56 \mathrm{~d}$ observed light curve decay rate. The two parameters that determine the deposition modification are: (1) the time necessary to expand to a thickness of $\tau=1$ $\left(t_{\text {expan }}=1\right)$ and $(2)$ the radioactive decay time $\left(t_{\text {half }}\right)$ where we are using the radioactive half life to more easily scale to the familiar numbers $77 \mathrm{~d}$ and $56 \mathrm{~d}$. The ratio, $t_{\text {expan }} / t_{\text {half }}=R_{\text {expan/half }}$ determines the luminosity (or deposition fraction) versus $t_{\text {half }}$. The set of modified decay curves for various ratios, $R_{\text {expan/half, }}$ versus time in units of $t_{\text {half }}$ are shown in Fig.4. 
The fractional deposition, eq. (7), is shown on the opposite axis. One sees that when the ratio is large, one recovers the original radioactive decay rate, or equivalently, $D_{\text {dep }}=1$. However, when the ratio is small, corresponding to rapid expansion, the decay of the deposited energy is more rapid than the radioactive decay itself.

We now wish to relate $R_{\text {expan/half }}$ to the various models. In CPR we expressed the characteristic evolution of the thickness of the various models in terms of the parameter $\left(M_{e j} / v_{9}^{2}\right)$. The transparency time then becomes $t_{\text {expan }}=42\left(M_{e j} / v_{9}^{2}\right)^{1 / 2} \mathrm{~d}$ for gamma-rays in a nebula of silicon burning products and $790\left(M_{e j} / v_{9}^{2}\right)^{1 / 2} \mathrm{~d}$ for the betas. The corresponding ratios become $R_{\text {expan } / \text { half }}=0.54\left(M_{e j} / v_{9}^{2}\right)^{1 / 2}$ and $=10.2\left(M_{e j} / v_{9}^{2}\right)^{1 / 2}$ respectively. In order to fit the observed 56 day half life according to the straight line of this slope in fig. 4, we require $R_{\text {expan/half }} \sim 4$ corresponding to $M_{e j} / v_{9}^{2}=$ 0.16 . We have used $M_{e j} / v_{9}^{2}=0.15$ for our low mass single zone model that gives the best fit to the data. For the gammas this gives $t_{\text {expan }}=16 \mathrm{~d}$ or $R_{\text {expan } / \text { half }} \sim 0.2$. This decay is too rapid to be approximated by a modified exponential, but the single zone model calculations show good agreement with the observations of rapid decay of the luminosity after peak for the "fast" S:IIAs due to the rapid gamma-ray transparency.

\subsection{THE LATE-TIME LIGHT CURVE}

The primary assumption of the late-time light curve (after peak luminosity) is that all the deposited radioactive energy is re-radiated. The major fraction of this energy is radiated in the visible part of the spectrum, because in order for atomic states to be produced, excited, and then radiate sufficient energy, a fraction of some of the most abundant elements must be ionized. Because the lowest ionization potential of the abundant elements are roughly $\gtrsim 6 \mathrm{eV}$, partial ionization at very low density requres $T \gtrsim 0.5$ $\mathrm{eV}$, or $6000 \mathrm{~K}$. The characteristic emission of the thermal electrons is then in the visible part of the spectrum where the radiation can escape. Radiation is also emitted in the UV and IR by excited states. When high energy particles, Compton electrons or positrons dissipate their energy in a plasma, a major fraction of the ionization energy flows through hard UV photons. These UV photons are readily absorbed in the nebula and may either heat the background electron plasma, or may be absorbed by an atom leaving it in an excited state from which it decays preferentially through several lower energy transitions. This latter process is fluorescence, and for a UV photon usually means that the several decay states lead to visible radiation. The nebula is either diffusively thin or transparent to the visible photons, which then escape freely. This is the mechanism invoked for the origin of the visible to blue radiation of the late time observations of 1972E by Kirshner 
and Oke (1975) where the fluorescent matter is singly ionized iron, FeII. This path for the ionization energy flow was neglected by Axelrod (1980, his fig. 1), but emphasized by Myerott (1980) and recently by Li and McCray (1995). Axelrod (1980), on the other hand, invoked a radiation flow directly from the thermal plasma through several unique low-energy states of Fell and therefore presumed that the radioactive energy was emitted in the IR. This emission in the IR was not only invisible to standard photon detectors, but also insured that the temperature of the plasma fell quickly below the point where visible levels would be excited by the thermal electrons, and hence, it was called the IR catastrophe. This would not affect the fluorescent radiation. These states are roughly at 100 micron wavelengths and were assumed to emit in the transparent limit for the calculation.

CPK rejected the IR emission as an explanation for the $56 \mathrm{~d}$ decay because (1) the fraction of the ionization loss through UV photons and processed by fluorscence should remain constant and not result in a progressively modified decay curve for the visible fraction and (2) the blackbody Wein limit at 100 microns was too small. The radiation flux in the IR is given by

$$
\phi(\nu)=\left(2 k T / c^{2}\right) \nu^{3}(\Delta \nu / \nu) \approx 4.8 \times 10^{4}(\Delta \nu / \nu) \operatorname{erg~} \mathrm{cm}^{-2} \mathrm{~s}^{-1} .
$$

Here $T=6000$, and the fractional width, $\Delta \nu / \nu$, of the lines is due to the velocity dispersion of the photosphere known as the Sobeloff width. For a photosphere velocity of $\sim 10^{9} \mathrm{~cm} \mathrm{~s}^{-1}$ and a density gradient of $\rho \propto R^{-7}$ then $\Delta \nu / \nu \sim 1 / 200$. The emitted flux per line becomes $\phi(\nu) \times 4 \pi R^{2}$. At the time when the luminosity decay departs from the expected $77 \mathrm{~d}$ beta decay by a factor of $\sim 1 / 2, t \sim 300 \mathrm{~d}$ (see Fig. 10), the IR luminosity would then have to be $L_{I R} \sim 5 \times 10^{39} \mathrm{ergs} \mathrm{s}^{-1}$. By contrast the total IR radiant energy per line by (9) should be limited to $L_{I R} \sim 2 \times 10^{36} \mathrm{ergs} \mathrm{s}^{-1}$. It is therefore very unlikely that any combination of lines near 100 micron wavelength can make up for the very large difference in these numbers. Furthermore, if the IR were effective in this fashion, it would lead to a very sudden "catastrophic" termination of the optical luminosity rather than the gradual decline described by the 56 day half-life. Finally no observations have been made in this wave length band with the required sensitivity to observe this emission (pvt. comm. Peter Meikle). It is for these reasons that CPK felt compelled to find an alternate explanation to modify the decay rate which led to mechanism requiring the transparency of the nebula to the positrons created in the minor branch of the $\mathrm{Co}^{56}$ decay.

\subsection{DUST}

Since the time of this conclusion, the nearby supernova, $1987 \mathrm{~A}$ occured with its asssociated emmense effort of observation and interpretation. Of course 
the expected and most gratifying observations were those of the neutrinos, the gamma-rays and $\mathrm{x}$-rays, and the $77 \mathrm{~d}$ half life of the initial luminosity decay curve confirming the dominant role of $\mathrm{Ni}^{56}$ in its formation. One of the surprises was the observation of 10 micron IR emission late in time by many observations and reviewd in Whitelock, Catchpole and Feast, (1991). This emission peaked at roughly $600 \mathrm{~d}$ and remained thereafter a constant fraction of near $30 \%$ of the optical luminosity. (It is worthwhile pointing out that in this interpretation the loss of energy by gamma transparency was negelected, see Fig. 6.) It was interpreted by Lucy et. al. \& Danziger,et al., (1991) as emission from dust grains formed in the expanding nebula, and the IR emission made a significant contribution to the more rapid decline (than 77d) of the light curve after $550 \mathrm{~d}$. Could this same processes of dust formation and IR emission be the explaination of the missing energy at late times in SNIAs? We do not believe that this is at all reasonable. Lucy et al. (1991 and ref. therein) calculated the grain formation in the expanding nebula and suggested a high efficiency, $\sim 1$, if the matter of the nebula is clumped. However, without clumping Hashimoto et al. (1989) calculate a maximum efficiency of $10^{-3}$ by day 775 . The growth of a dust grain depends upon the density of the matter and that lic teryurature is low enough to permit condensation of the molecules. This process does not start for 1987 A until day 500 and we expect a lower density and higher temperature for a Chandrasekhar-mass thermonuclear model. The density for the $1.4 M_{\odot}$ model should be $\sim 260 \times$ greater and the energy deposition per gram, (leading to heating and temperature) should be $\sim \times 20$ greater than 1987 A. Yet 1987 A produced grains necessary to account for the missing energy three times later than necessary to explain the missing energy of type IAs and furthermore was expected theoretically to lead to large clumping. It therefore seems unreasonable to invoke grain formation to explain the 56 d late light curve of SNIAs.

\subsection{POSITRON TRANSPARENCY}

The positron transparency explanation of the modified $56 \mathrm{~d}$ decay is based upon the assumption that a fraction of the positrons from the $\mathrm{Co}^{56}$ decay can escape the nebula. The reason for assuming that the energy emitted in the $56 \mathrm{~d}$ decay is derived from the deposition of the positrons rather than the gamma-rays is that the nebula at late times becomes too thin to capture sufficient energy from the gamma-rays. For example, in the W7 model the time to gamma-ray transparency as stated above is $t_{\gamma t r a n s}=41$. Since the positrons contribute $4.0 \%$ to the $\mathrm{Co}^{56}$ decay energy, the ratio of the thicknesses where the energy deposited by gamma-rays equals the energy deposited by the $4 \%$ positron fraction, using eq. (7), is $\tau_{\gamma} / \tau_{\beta}=$ 
$(0.64 / 0.04)=16$. Since $\tau \propto t^{-2}$, then the time when $\dot{W}_{\text {dep }}=\dot{W}_{\beta}$ is $t_{\beta \text { trans }}=4 \times t_{\text {trans }}=176$ days. In CPK, an analysis was made of positron (electron) range energy curves for the spectrum expected from the $\mathrm{Co}^{56}$ positron branch. Because of scattering and the beta decay energy distribution, this led to the rather surprising result that the number of positrons whose range exceeded a given thickness was exponential over a limited range in thickness just as it was for gamma-rays, but with a mean positron range $1 / 355$ that of the gamma-rays or $0.1 \mathrm{~g} \mathrm{~cm}^{-2}$. Thus the time to positron transparency would be 18.4 times that of the gamma-rays. When the nebula is thin to gamma-rays, it is thick to positrons and it takes a factor of $\sim 4 \times$ longer for the nebula to become transparent to positrons.

Of course even weak magnetic fields would confine such low energy particles, but CPK pointed out that the initial field of the pre-supernova star would be "combed" radially by the expanding matter and that a dipole field would give gyrating particles the same path length distribution as that for gamma-rays travelling in a straight line. As a consequence, we can use the same simple form of the deposition fraction as we used for the gammaray deposition [eq. (7)], except with an effective thickness 3.55 times greater.

Before discussing these calculations, we will first address the question of positron escape. It has frequently been pointed out that if a a positron were to escape the nebula, the external field lines would return the particle to the surface of the nebula and therefore it would not escape. Here we point out that the energy density of the escaping positrons, and even more so for the initial Compton electrons from the escaping gamma-rays, greatly exceeds, by many orders of magnitude, the energy density of the external magnetic field. Consider a strong initial field of $10^{7}$ gauss expanded after half a year to $10^{8}$ times the initial radius, or $2 \times 10^{16} \mathrm{~cm}$. The energy density of the field is reduced to $B^{2} / 8 \pi \sim 4 \times 10^{-20} \mathrm{ergs} \mathrm{cm}^{-3}$. The energy of the original expanding Compton electrons will be $\sim(1 / 10)\left(1-\dot{W}_{\text {dep }}\right) t_{\text {decay }} \sim 10^{48}$ ergs, and the energy density, assuming a relativistic expansion at a velocity of $c / 3$, will be $\sim 10^{48} /(4 \pi / 3)\left[(c / 3) 1.5 \times 10^{7}\right]^{3} \sim 7 \times 10^{-4} \mathrm{erg} \mathrm{cm}^{-3}$. Thus both the escaping Compton electron pressure and the positron pressure will greatly exceed the confinement pressure of the magnetic field. Therefore the magnetized relativistic gas will expand freely and all the relativisitic particles will escape freely into the ISM.

There still remains, however, the question of whether the internal turbulence in the expanding nebula as interpreted for 1987A might wrap up the magnetic field sufficiently to confine the positrons. The instabilites interpreted from the optical, gamma-ray, and x-ray results depend upon the explosion shock running into a larger envelope mass, being decelerated, and causing Raleigh-Taylor instabilities. Since there is no massive envelope in the following low mass models of Type Ias, there is no apparent way to 
create the necessary instabilities to confine the positrons. Finally we note that the maximum initial magnetic field such that positrons would escape independent of the field is very low, which means that if there were turbulence in the expanding debula, positrons would be confined. Assuming $R_{L} \sim(1 / 3) R_{t}$ where $R_{L} \sim \frac{2 \times 10^{3} \text { gauss cm }}{B}$ is the Larmor orbit (aka gyration radius) of a typical positron and $R_{t} \sim 2 \times 10^{16} \mathrm{~cm}$ is the radius of the nebula after $0.5 y$. Then for these parameters $B_{\text {initial }} \leqslant 3000$ gauss, a field small compared to the initial field, $10^{7}$ gauss, necessary to create a standard, $10^{12}$ gauss magnetized neutron star by flux conserving collapse. Assuming the escape of the positrons is the only reasonable explanation for the 56 day light curve, and that therefore turbulence or mixing is low in these low mass models, we then consider and interpret the calculations using the single zone model.

\section{The Single Zone Models}

\section{1. $1987 \mathrm{~A}$}

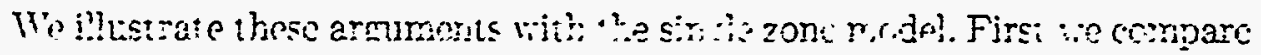
the model to the observations of $1987 \mathrm{~A}$ where, due to the large mass and slower expansion velocity, neither the enhancement opacity nor positron transparency are an issue.(However, the gamma-ray transparency does result in a similar modification of the late light curve.) We have therefore used a constant optical opacity, $0.045 \mathrm{~cm}^{2} \mathrm{~g}^{-1}$. Fig. 5 shows the light curve for the first 200 days, which gives the maximum at roughly the correct time (90 days). However, the observations at maximum rise a factor of $\sim 2$ above the radioactive deposition energy in contrast to the approximation of the single zone model. The reason for this difference is that a significant fraction of the internal energy is stored in latent heat of ionization for this high density model and is released at the time of maximum. It is less important in the low mass models. In a short period after maximum the observed light curve declines to match the radioactive decay energy with $100 \%$ deposition. The calculated light curve both with and without the deposition fraction are the same at early times as expected. The positron energy in this case is negligible. Fig. 6 shows the late-time light curve of 1987A.

First, and most important, we observe that when the deposition fraction (of the gamma-rays) is predicted to be $100 \%$, the light curve follows the $77 \mathrm{~d}$ half life as it should. (The 77d half life decay of the luminosity of $1980 \mathrm{k}$, another type II SN was observed earlier by Uomoto and Kirshner, (1985).) Secondly when gamma-ray transparency starts at $\sim 450 \mathrm{~d}$, the luminosity including the deposition fraction falls below the case with $100 \%$ deposition. In this model, gamma-ray transparency occurs at roughly $450 \mathrm{~d}$, so that $R_{\text {expan } / \text { half }}=5.7$ with a resulting modified half life of $65 \mathrm{~d}$ from Fig. 3 . This 
matches the synthsized bolemetric light curve of Woosley. Here, however, infared radiation ( $~ 2$ to 10 microns) must be included and it does affect the luminosity in the visible band as it becomes roughly half of the luminosity at $600 \mathrm{~d}$. This IR luminosity is at shorter wavelengths, $\sim 10$ microns, than the expected Fell emission, avoiding the Wein limit. The grain emission would not be expected for the low mass models as discussed in the text. In this case the modification of bolemetric luminosity decay rate is due to both the transparency to the gamma-rays as well as IR, but for the low mass models we invoke transparency to the positrons. Here positrons make only a small contribution to the light curve, because the gamma-rays are nearly all deposited as well. The single zone model gives a simple interpretation of the time of the peak and the late light curve. The next step is to apply these approximations including the enhancement opacity to reproduce the numerical calculations by Harkness (1991) of the theoretical W7 model of Nomoto.

\subsection{W7}

We have already used plots of the internal energy vs. time in fig. 1 to demonstrate the energy effects of adiabatic expansion, radioactive decay energy, and diffusion. Fig. 4 shows the deposition fraction of gamma-rays in the single zone model as derived from Eqs. $7 \& 8$ and the calculations from Harkness (1991). Fig. 7 shows the single zone calculations of the W7 model of Nomoto (1984) for the first 50 days. Both enhanced (expansion) opacities and constant opacities $\left(\kappa=0.1 \mathrm{~cm}^{2} \mathrm{~g}^{-1}\right)$ are modeled, and as expected, the expansion opacity delays the maximum luminosity from $12 \mathrm{~d}$ to $16 \mathrm{~d}$ and reduces the maximum from $1.5 \times 10^{43}$ to $1.15 \times 10^{43} \mathrm{erg} \mathrm{s}^{-1}$. The calculations are compared to the multi-zone calculation of Harkness (1991) with satisfactory agreement thus substantiating the single zone approximations. The calculations are also compared to observations where the first data point of $1972 \mathrm{E}$ (Kirshner and Oke, 1975) is chosen to be just after maximum (17d), and Leibundgut's (1991) template of many supernovae which is matched only at the maximum luminosity. However, both calculations together are significantly above the late-time observations for $t>30 \mathrm{~d}$. This would indicate that after light maximum, where the luminosity is entirely due to the gamma-ray energy deposition rate, that the transparency due to gamma-rays is greater than the calculations by a significant factor. Greater transparency requires either less mass or greater velocity.

At late times (Fig. 8) this indication of too great a thickness, or too small a transparency is equally apparent for the particular SN1972E. This same light curve, the straight line superimposed upon the Kirshner points, has been observed for 1992A (Kirshner, this conference and Suntzeff, 1994). 
Although these two supernova may be exceptionally "fast" in their decline, they strongly suggest a requirement for smaller mass and a higher mass ejection velocity than the $1.4 M_{\odot}$ models.

\subsection{LOW MASS}

The parameters chosen for the best fit to the observations of $72 \mathrm{E}$ and $92 \mathrm{~A}$ both early as well as late time decay are $0.4 M_{\odot}$ ejected with 0.8 foe $\left(8 \times 10^{50}\right.$ ergs) with $0.35 M_{\odot}$ of $\mathrm{Ni}^{56}$ (figs. 9 and 10). The mass of $\mathrm{Ni}^{56}$ is somewhat arbatrary and determines the peak luminosity (and hence the Hubble constant), $5 \times 10^{42}$ ergs $\mathrm{s}^{-1}$ and influences the spectral fit. The parameter proportional to the transparency (see CPK) is $M_{e j} / v_{9}^{2}$ with values $0.15,1.13, \& 105$ for the respective models of low mass $\left(0.4 M_{\odot}\right), W 7$, $\left(1.4 M_{\odot}\right)$, and $1987 \mathrm{~A},\left(14.5 M_{\odot}\right)$ respectively. In CPK the same transparency parameter for the low mass interpretation was estimated to be 0.22 , somewhat higher than the present low mass model. One also observes that here the expansion opacity makes a large difference such that the time to maximum light is extended from 6 days with a constant opacity, $\kappa=0.2 \mathrm{~g} \mathrm{~cm}^{-2}$ to 13.5 days with the expansion opacity. This is the effect that compersa. es the time to maximum light for the greatly differing expansion factors. One notes that the last point of $1972 \mathrm{E}$ at $722 \mathrm{~d}$ falls a factor of 8 below the beta energy curve indicating that this much energy had to be lost from the observations. If one assumed that the gamma-rays were also absorbed, then this point falls a factor 200 below the gamma-ray curve. However, it is not just that a single point falls so far below the beta energy rate, but the near constant exponent of the exponential late-timelight curve that indicates a simple physical process. It is for these reasons using our simple single zone model, that we believe that a low mass model of type $1 \mathrm{~A}$ supernovae makes better sense than the ejection of a much larger Chandrasekhar mass.

\subsection{SPECTRA}

There remains the caveat that this analysis does not address spectra, and indeed the spectral fits offer a strong constraint, but the expansion opacity effect should make the spectra less dependent upon mass since the photosphere must reside at a slightly smaller radius and a higher velocity with the same temperature to result in nearly the same time to maximum for the two models, W7 and low mass. The excitation of the the same elements (silicon burning) with the same temperature, velocity, and velocity dispersion will tend to create similar spectra regardless of the mass involved. The mass determines the optical depth, but as we have shown previously, the effects of the expansion opacity severely limit even this dependence upon mass. 


\section{Possible Supernova Mechanisms}

Driven by the late-time light curves to low mass ejection for some subclass of Type Ias, we will now discuss the possible mechanisms for low-mass Type Ia supernovae. A succesful mechanism must satisfy three requirements: 1) the progenitor of the mechanism must occur at rates comparable to the supernova rate, 2) the mechanism must explode (that is, the mechanism must eject sufficient material (greater than $\sim 0.1 M_{\odot} \mathrm{Ni}$ ) at supernova energies ( $10^{51} \mathrm{ergs}$ ), and finally, 3 ) the mechanism must have nucleosynthetic yields that satisfy spectral constraints and the constraints set by galactic nucleosynthesis. Two mechanisms present themselves as viable choices: sub-Chandrasekhar mass thermonuclear explosions (SCTs) and accretion induced collapse of white dwarfs (AICs).

\subsection{SUB-CHANDRASEKHAR MASS THERMONUCLEAR EXPLOSIONS}

One of the most tantalizing aspects of SCTs is the large occurrence rate of their progenitors. The two main progenitors involve either $\mathrm{CO}$ white dwarfs accreting helium from nondegenerate companions that are burning helium in their core (Iben \& Tutukov 1991, Limongi \& Tornambe 1991) or hot CO white dwarfs accreting from red giant companions, also known as symbiotic systems (Munari \& Renzini 1992, Yungelson et al. 1995). Tben and Tutukov predict a formation rate $\sim 0.01 \mathrm{y}^{-1}$ which is comparable (within an order of magnitude) to the rates given by Yungelson et al. for symbiotic systems. These rates can comfortably account for the type Ia supernova rate.

Livne (1990) and Livne \& Glasner (1991) have demonstrated the success of the SCT mechanism in both one and two dimensions. The simplistic picture behind their model is the following: Helium is accreted onto the white dwarf until temperatures near the base of the helium layer are sufficient to ignite the helium (with the aid of a $\mathrm{N}$ spark). The helium detonation compresses the $\mathrm{CO}$ core, ultimately causing its detonation and the resultant supernova explosion. Recent results by Arnett (1995) and Benz (1995) have cast some doubt upon the robustness of this mechanism, mandating a more complex picture. The success of Livne and Livne \& Glasner prompted Woosley \& Weaver (1994) to run a series of one-dimensional SCT models predicting a range of nickel mass ejection $\left(0.2-0.9 M_{\odot}\right)$, total mass ejection $\left(0.8-1.1 M_{\odot}\right)$ and kinetic energies $\left(0.8-1.4 \times 10^{51} \mathrm{ergs}\right)$.

Woosley \& Weaver also conducted preliminary analyses of light curves (up to 100 days) and nucleosynthetic yields under this mechanism. The light curves are quite reasonable and their variations may provide an ideal explanation for the observed variations in type Ia light curves. Their nucleosynthetic yields, beyond an overproduction of ${ }^{44} \mathrm{Ti}$ and other isotopes of 
$\mathrm{Ti}, \mathrm{Cr}$, and $\mathrm{V}$, are reasonable. However, as Woosley \& Weaver point out, much more detailed simulations are required to verify these results.

\subsection{ACCRETION INDUCED COLLAPSE}

Much effort has been put into studying the progenitors for AICs (see Canal, Isern, \& Labay 1990 for a partial review). The rate of formation and evidence for these progenitors grows with the list of publications. CO and ONe white dwarfs pushed beyond the Chandrasekhar mass limit will either explode thermonuclearly or collapse into a neutron star. Although the fate of these accreting white dwarfs can be determined by the accretion rate and initial white dwarf mass (Nomoto \& Kondo 1991), the results depend sensitively upon the input physics (URCA processes, screening effects, etc.) Assuming ONe white dwarfs to be the sole contributor to AICs, Tben, Tutukov, \& Yungelson predict an AIC rate of $3 \times 10^{-4} \mathrm{y}^{-1}$ in the galactic disk, and Bailyn and Grindlay (1990), in an effort to explain the millisecond pulsar population in globular clusters, require a comparable rate $\left(\sim 10^{-4} \mathrm{y}^{-1}\right)$ in. the grlactic globular cluster population. Although these numbers will vary witl the inpul physics, the totai rate of white dwaris being pushed over the Chandrasekhar limit is generally calculated to be too small to explain all type Ia supernovae. White dwarf collisions are also thought to result in AICs (Mochkovitch \& Livio 1990) but they occur rarely and will not improve the rates. However, these low rates are even more acute for the current "standard" mechanism for type Ia: Chandrasekhar-mass thermonuclear explosions.

Despite the immense work studying the progenitors of AICs, very little work has been devoted to the mechanism driving their explosion. Most of the work (e.g. Baron et al. 1987) modeled the collapse through the stall of the bounce shock verifying only that the "prompt" mechanism was insufficient to induce a Type Ia supernova explosion, just as it is incapable of powering a Type II explosion. Mayle \& Wilson (1988) studied the collapse of OMgNe cores of stars within the mass range $8 M_{\odot}<M<10 M_{\odot}$ which have structures similar to AIC progenitors. By following the late-time neutrino heating which has become so central to the type II mechanism, Mayle $\&$ Wilson found that an explosion occurred ejecting $0.042 M_{\odot}$ with energies in the range $0.6-1.2 \times 10^{51}$ ergs. Woosley \& Baron (1992 - hereafter WB), using a AIC progenitor from Nomoto (1986), ran a complete caclulation of the collapse resulting in no mass ejected under the delayed neutrino mechanism. However, a neutrino wind did eject $\sim 0.005 M_{\odot}$ at energies around $0.5 \times 10^{51}$ ergs. This wind, however, would most likely be present in the Mayle \& Wilson simulation if they had continued it for several seconds with sufficient resolution. 
We have run a series of simulations using the Nomoto progenitor and have found a strong dependence of the ejected material upon the implementation of the neutrino physics (source terms, flux limiter, etc.) and the equation of state (Fryer et al. 1995). Our simulations lead to mass ejecta up to $\sim 0.2 M_{\odot}\left(\sim 0.1 M_{\odot} \mathrm{Ni}\right)$ with kinetic energies reaching $\sim 10^{51} \mathrm{ergs}$. (Figure 11 displays for the mass-point trajectories over the course of a typical simulation.) Although we have not calculated spectra of these explosions, the nucleosynthetic yields already pose a problem with a large amount $\left(\sim 0.1 M_{\odot}\right)$ of neutron rich material $\left(Y_{e}<0.45\right)$, producing anomolously high abundances of unwanted species such as ${ }^{88} \mathrm{Sr}$. The electron fraction of this material is also sensitive to the neutrino fraction however, and this problem may be solved by corrections in the neutrino physics or the addition of neutrino oscillations(Fuller and Meyer 1994). Because these results are significantly different from the WB results, we will concentrate the next few paragraphs on the pitfalls and problems involved with these calculations.

Neutrino transport has attracted much attention over the past few years (ค. Jarka 19?2) in modeling tyne II supernciae. The difficulty arises

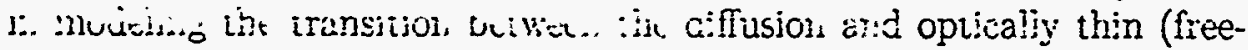
streaming) regimes. Typically, a flux limiter is invoked to connect these two limits. We have implemented several flux limiters (Levermore \& Pomraning 1981, Bowers \& Wilson 1982, Herant et al. 1994) which bracket the more accurate calculations of Janka (1992). These do vary the mass ejecta ( $50 \%$ ) but do not account for the differences in our results from those of WB.

Significantly less emphasis has been placed upon the neutrino source terms themselves. Often times, assumptions are made to simplify the numerics of the source terms. In most analytic estimates shown in the literature, degeneracy is ignored when determining the neutrino emission. WB make a different assumption that the emitting electrons are highly relativistic. Both these assumptions could have drastic effects both on AICs and other applications involving neutrino emission.

An accurate numerical representaion of the electron/positron neutrino emission rates and luminosity has been derived by Takahashi, El Eid \&Hillebrandt 1977:

$$
\begin{aligned}
& \lambda_{\substack{e^{-p} \\
e^{+n}}}=C_{2} \beta^{-5}\left[F_{4}( \pm \eta) \pm(2-2 \Delta) F_{3}( \pm \eta) \beta+\right. \\
& \left(\frac{1-8 \Delta+2 \Delta^{2}}{2}\right) F_{2}( \pm \eta) \beta^{2} \\
& \pm\left(2 \Delta^{2}-\Delta\right) F_{1}( \pm \eta) \beta^{3}+
\end{aligned}
$$




$$
\left.\left(\frac{4 \Delta^{2}-1}{8}\right) F_{0}( \pm \eta) \beta^{4}\right]
$$

and

$$
\begin{array}{r}
L_{\bar{\nu}}=C_{3} \beta^{-6}\left[F_{5}( \pm \eta) \pm(2-3 \Delta) F_{4}( \pm \eta) \beta+\right. \\
\left(\frac{1-12 \Delta+6 \Delta^{2}}{2}\right) F_{3}( \pm \eta) \beta^{2} \\
\pm\left(\frac{-3 \Delta \pm 12 \Delta^{2}-2 \Delta^{3}}{2}\right) F_{2}( \pm \eta) \beta^{3}+ \\
\left(\frac{-1+12 \Delta^{2}-16 \Delta^{3}}{8}\right) F_{1}( \pm \eta) \beta^{4} \\
\left. \pm\left(\frac{2+3 \Delta-4 \Delta^{3}}{8}\right) F_{0}( \pm \eta) \beta^{5}\right]
\end{array}
$$

vitiere $\lambda_{e^{-p}}$ are the transition rates for eleciron ard positron capture, $I_{\bar{\nu}}$ are the electron neutrino and anti-electron neutrino luminosities, $C_{2}=6.15 \times$ $10^{-4} \mathrm{~s}^{-1}$ per nucleon, $C_{3}=5.04 \times 10^{-10} \mathrm{erg} \mathrm{s}^{-1}$ per nucleon, $\Delta=1.531$ is the neutron-hydrogen mass difference and $\beta=\frac{m_{c} c^{2}}{k_{k T} T} . F_{n}$ are fermi integrals of order $\mathrm{n}$ and $\eta$ is the degeneracy parameter. WB have simplified these equations, taking only the first term in each equation. Figure 12 illustrates the difference in average neutrino energy from this simplication and the full equation for a range of degeneracy parameters $\eta$. For typical values in our simulations $\left(\eta=4, T_{\text {gas }}=2 \times 10^{10} \mathrm{~K}\right)$, the difference in average electron energy is nearly $30 \%$ which corresponds in a decrease in neutrino heating by $70 \%$. Note also the strong dependence upon the degeneracy parameter. For large $\eta, F_{5}(\eta) \propto \eta^{6}$ and the neutrino luminosity depends on temperature and degeneracy to the same power. Lowering the temperature increaseses the degeneracy and the subsequent neutrino luminosity may remain stable!

However, the primary difference between our results and those of WB rests in the equation of state. Two dense equations of state have been commonly used in type II collapse scenarios: SL (developed by Lattimer \& Swesty 1991) and BCK (see Baron, Cooperstein, \& Kahana). We have varied many of the equation of state parameters in the BCK EOS and the differences in the results remain. In all the simulations using the BCK EOS, very little matter, if any, is ejected under either the prompt or delayedneutrino explosion mechanism. As with WB, the only ejecta is in the neutrino wind at later times. With all of the SL EOS simulations, between $0.08-0.2 M_{\odot}$ of material is ejected with energies ranging from $0.1-1.5$ foe. 
As discussed in (Swesty, Lattimer, \& Myra 1994), the SL equation of state is considered to be more reliable and we used the SL EOS simulations in our above analysis. However, all of these uncertainties must be sorted out to make any firm conclusions.

\subsection{MULTIPLE MECHANISMS}

Peculiar objects such as $1991 \mathrm{bg}$, which is both underluminous and has odd spectral features with respect to "standard" type Ias (Filippenko et al. 1992), suggests that there may be more than one mechanism behind type Ia supernovae. Therefore, a combination of the above mechanisms may be required to explain all the observations.

One can differentiate between AICs and SCTs directly by the neutrino detection of nearby Type las or by finding neutron star remnants from Type Ia explosions. Beyond this direct evidence, one may also form an indirect test by measuring masses of neutron stars in systems whose evolution involved a past AIC event ( $R$. Canal - pvt communication). If the AIC is indeed shedding $0.2 M_{\odot}$ then the neutron stars in these systems should be less massive than the "typical" neutron stars formed in type II explosions.

\section{Acknowledgements}

This paper has benefitted from many discussions; before, during and at this NATO ASI meeting on Type I supernovae. We would like to thank Ed Baron, Robert Harkness, Peter Höflich, Albert Petschek, Craig Wheeler, and Stan Woosley for both helpful discussions and for providing us with their simulations. The work and travel of C.F. was funded by NSF grant AST 9206738, LANL GRA program, NSF travel awards, and the NATO ASI program. The work of S.A.C. has been supported by the DOE.

\section{References}

Arnett, W.D., 1982, ApJ, 253, 785

Axelrod, T.S., 1980a, Texas Workshop on type I Supernovae, Austen Texas, Craig Wheeler, ed., 80.

Axelrod, T.S., 1980b, Ph.D. Thesis, Univ. Calif. Santa Cruz, UCRL52994

Baade,W. and Zwicky, F., 1938, ApJ, 88,411

Brade, W., Burbidge, G.R., Fowler, F., Burbidge, E.M., Christy, R.F., and Fowler, W.A., 1956, Pub. A.S. P., 68, 296

Bailyn, C.D., Jonathan E.G. 1990, ApJ, 353, 159

Baron, E., Cooperstein, J., Kahana, S., Nomoto, K. 1987, ApJ, 320, 304

Bowers, R.L. \& Wilson, J.R., 1982, ApJS, 50, 115

Burbidge, E.M., Burbidge, G.R., Fowler, W.,Hoyle, F., 1957, Rev. Mod. Phys.29, 547

Canal, R., Isern, J., Labay, J. 1990, Annu. Rev. Astron. Astrophys., 28, 183

Colgate, S.A. \& McKee, R.H., 1969, ApJ,157, 623

Colgate, S.A. \& White, R.H., 1966, ApJ,143,626 
Colgate, S.A., Petschek, A.G., \& Kriese, J.T., 1980, ApJ, 237, L81

Colgate, S.A. \& Petschek, A.G. 1980, Texas Workshop on type 1 Supernovae, Austen Texas, Craig Wheeler, ed., 42.

Colgate, S.A., Petschek, A.G., 1982, Nature, 296, 804

Colgate, S.A., 1991, "Supernovae", Stan Woosley ed. Tenth Santa Cruz Workshop, Springer-Verlag, NY, 352

Danziger, I.J., Lucy, L.B., Bouchet, P., \& Gouiffes, C., 1991, "Supernovae", Stan Woosley ed. Tenth Santa Cruz Workshop, Springer-Verlag, NY, 69

Dogget, J.B. and Branch, D., 1985, Astron.J., 90, 2303

Filippenko, A.V., Richmond, M.W., Branch, D., Gaskell, C.M., Herbst, W., Ford, C.H., Treffers, R.R., Matheson, T., Ho, L.C., Dey, A., 1992 AJ 1041543

Fryer, C.L., Herant, M., Colgate, S.A., Benz, W. 1995 in preparation

Fuller, G.M., \& Meyer, B.S. 1994, preprint

Harkness, R., 1991, "Supernovae", Stan Woosley ed. Tenth Santa Cruz Workshop, Springer-Verlag, NY

Hashimoto, M., Nomoto, T., \& Shigeyama, T., 1989, Astr. Ap. 210 L5 Y

Herant, M., Benz, W., Hix, W.R., Fryer, C., Colgate, S.A., 1994, ApJ, 435, 339

Höflich, P., Khokhlov, A., and Wheeler, J.C., 1995, ApJ., in press

Höflich, P., 1990, Habilition Thesis, Ludwig Maximilians Univ., Munchen,pub. as MaxPlank-Inst. für Astrophysik 1990, Preprint 563

Iben, I., Tutukov, A.V. 1991, ApJ, 370, 615

Iben, I., Tutukov, A.V., Yungelson, L.R. 1995, submitted to ApJ

Janka, H., 1992, A\&A, 256, 452

Karp, A.H., Lasher, G., Chan, K.L., and Salpeter, E.E., 1977, ApJ., 214, 161

Kirshner, R.P., 1990, "Supernovae", ed. Albert Petschek, Springer-Verlag, 59

Kirshner, R.P. and Oke, J.B., 1975, ApJ., 200,574

Lattimer, J.M., Swesty, F.D., 1991, Nucl. Phys. A, 535, 331

Levermore, C.D., \& Pomraning, G.C., 1981, ApJ, 248, 321

Li, H. and McCray, R., 1995, "UV Opacity and Fluorescence in SN Envelopes" JILA, Prepint 253, and ApJ submitted

Limongi, M., \& Tornambe, A. 1991, ApJ, 371, 317

Livne, E. 1990, ApJ, 354, L53

Livne, E. \& Glasner, A.S. 1991, ApJ, 371, 317

Lucy, L.B., Danziger, I.J., Bouchet, P., \& Gouiffes, C., 1991, "Supernovae", Stan Woosley ed. Tenth Santa Cruz Workshop, Springer-Verlag, NY, 82

Mayle, R., \& Wilson, J.R. 1988, ApJ, 334, 909

Minkowski, R., 1964, Ann. Rev. Astr. Ap. 2, 267

Mochkovitch, R. \& Livio, M., 1990, A\&A, 236, 378

Munari, U., \& Renzini, A. 1992, ApJ, 397, L87

Nomoto, K. 1986, Ann. New York Acad. Sci, 470, 294

Nomoto, K. \& Kondo, Y., 1991, ApJ, 367, L19

Nomoto,K., Thielemann, F.-K., and Yokoi, K., 1984, ApJ, 286, 644

Sobolev, V., 1960, In"Moving Envelopes of Stars", Harvard Univ., Cambridge

Swartz, D.A., Sutherland, P.G., \& Harkness, R.P., 1995, ApJ, 446, 766

Swesty, F.D., Lattimer, J.M., Myra, E.S., 1994, ApJ, 425, 195

Takahashi, K., El Eid, M.F., Hillebrandt, W. 1977, A\&A, 67, 185

Uomoto and Kirshner, R.,1985, ApJ, 308, 685

Weaver, T.A., Axelrod, T.S.,\& Woosley, S.E., 1980, Texas Workshop on type 1 Supernovae, Austen Texas, Craig Wheeler, ed., 80.

Wheeler, J.C., Harkness, R.P., and Khokhlov, A.M., 1995, Phys. Rpts., 256,211

Whitelock, P., Catchpole, R., and Feast, M. 1991, "Supernovae", Stan Woosley ed. Tenth Santa Cruz Workshop, Springer-Verlag, NY, 15

Woosley, S.E., \& Baron, E. 1992, ApJ, 391, 228

Woosley, S.E., \& Weaver, T.A. 1994, ApJ, 423, 371

Yungelson, L., Livio, M., Tutukov, A., \& Kenyon, S.J. 1995, ApJ, 447, 656 
Zwicky, F., 1938, Phys. Rev. 53, 1019 
Figure Captions

1. shows internal energy vs. time for the single zone model simulation of the conditions of the W7 Model. The curves in ascending order are: (1) the adiabtic expansion of the shock deposited energy $\left(E_{\text {int }}\right)$ with the expected slope of $-1,(2)$ with deposited gamma-ray and beta energy minus diffusion, (3) with deposited gamma-ray and beta energy without diffusion, (4) with all the $\mathrm{Ni}^{56}$ energy deposited.

2 . shows the expansion opacity function, $\kappa_{T}$, vs. temperature. The actual opacity becomes (opacity function) $\times$ (enhancement factorO, Eq. 4 .

3. shows deposition-modified exponential decay curves where the deposited energy or luminosity is $\dot{W} D_{\text {dep }}$. Time is in units of the decay time of the exponential, $t_{\text {decay }}$. The various curves are labeled by the time necessary to expand to a thickenss of unity, $t_{\text {expan }}$. The parameters for the various curves are the ratios, $R_{\text {param }}=t_{\text {expan }} / t_{\text {decay }}$. When $R_{\text {param }}$ is very large, one recovers the original exponential decay, but as $R_{\text {param }}$ becomes small, the effective decay of the deposited energy becomes faster, $\propto\left(t / t_{\text {expan }}\right)^{-2}$.

4. shows the deposition fraction vs. time for the W7 model using the analytic formulation of CPK (Petschek) in the single zone model (solid line) and the calculations of Harkness (1991) (dashed line) over the critical range of times where we migh expect the biggest deviation due to our single zone assumptions. In the region of transparency, eq. (8) agrees quite well (within the calculated errors) with the work of Swartz et al.

5 . shows the single zone model of 1987A for the first 200 days where the opacity is a constant, $0.045 \mathrm{~cm}^{2} \mathrm{~g}^{-1}$ as if the hydrogen were $24 \%$ ionized. The remaining parameters are $M_{\text {ejec }}=14.5 M_{\odot}, M_{N i}=0.078 M_{\odot}, E_{K}=$ 1.5 foes, $R_{\text {initial }}=2 \times 10^{12} \mathrm{~cm}$, and derived quantities $M_{\text {ejec }} / V_{9}^{2}=105$ and $V_{o}=3.72 \times 10^{8} \mathrm{~cm} \mathrm{~s}^{-1}$. The observational visual luminosity is by Catchpole et. al., 1987 etc. and the bolemetric light curve is synthesized by Woosley, 1988. The energy input is shown for gamma-rays and positrons separately and the derived luminosity is shown with diffusion and with and without deposition.

6. shows the single zone model of $1987 \mathrm{~A}$ for 1000 days with the same parameters as Fig. 5. The large thickness due to the larger mass and slower velocity means that initially all the gamma-rays are absorbed and thus one expects and the data confirms that the luminosity decay shortly after maximum follows the $77 \mathrm{~d}$ half-life of the $\mathrm{Co}^{56}$ decay. Thus all the deposited energy from radioactivity is emitted in the optical band. However, one notes the the maximum luminosity is significantly greater than the rate of deposited energy. This is well recognized as the storage and release of the heat of ionization. It is a smaller fraction in the faster, small-mass models. One also notes that the gamma-ray deposition modified decay after $\sim 400 \mathrm{~d}$ results in a modified decay of the approximately $65 \mathrm{~d}$ half-life. This curve 
agrees with the Woosley bolemetric curve including dust as compared to the catchpole observations where no correction has been made for possible missing IR due to dust emission.

7. shows single zone calculations of the W7 model of Nomoto (1984) for the first 50 days. Both enhanced or expansion opacity and constant opacity $\left(\kappa=0.1 \mathrm{~cm}^{2} \mathrm{~g}^{-1}\right)$ are modeled and, as expected, the expansion opacity delays the maximum luminosity from $12 \mathrm{~d}$ to $16 \mathrm{~d}$ and reduces the maximum from $1.5 \times 10^{43}$ to $1.15 \times 10^{43} \mathrm{erg} \mathrm{s}^{-1}$. The calculations are also compared to observations where the first data point of $1972 \mathrm{E}$ (Kirshner and Oke, 1975) is chosen to be just after maximum at 17d, and Leibundgut's template of many supernova (Leibundgut 1991) is matched only at the maximum luminosity. The parameters of this model are: $M_{e j e c}=1.40 M_{\odot}$, $M_{N i}=0.6 M_{\odot}, E_{K}=1.3$ foes, $R_{\text {initial }}=2 \times 10^{8} \mathrm{~cm}$, and derived quantities $M_{e j e c} / v_{9}^{2}=1.13$ and $v_{o}=1.11 \times 10^{9} \mathrm{~cm} \mathrm{~s}^{-1}$.

8. shows the same single zone calculations as figure 7 for 1000 days. The calculation after peak is just due to deposition, and one sees that transparency to betas occurs at 500 days (the intersection with the luminosity and $\dot{W}_{\beta}$ ), but it occurs too late in time as was the gamma-ray transprency in Fig. 7 to give agreement with the observations of 1972E. Kirshner has pointed out that the 1972E data agree well with the CTIO data of $1992 \mathrm{~A}$, (this conference, the solid straight line, Suntzeff, 1994).

9. shows a low mass single zone calculation where the mass and energy have been reduced to the parameters $M_{e j e c}=0.40 M_{\odot}, M_{N i}=0.35 M_{\odot}$, $E_{K}=0.8$ foes, $R_{\text {initial }}=2 \times 10^{8} \mathrm{~cm}$, and derived quantities $M_{e j e c} / v_{9}^{2}=0.15$ and $v_{0}=1.64 \times 10^{9} \mathrm{~cm} \mathrm{~s}^{-1}$. A constant opacity, $\kappa=0.2 \mathrm{~cm}^{2} \mathrm{~g}^{-1}$ and the expansion opacity have been used. The constant opacity case, as if all the matter were ionized, gives the maximum luminosity too early at 6 days. The expansion opacity case is significantly delayed to 13.5 days.

10. shows the low mass model at late times where the luminosity is matched to the $1987 \mathrm{E}$ data. The straight line simulating the $1992 \mathrm{~A}$ data discussed in Fig. 8 is not shown because it falls identically on top of the single zone approximation.

11. shows the mass point trajectories for a AIC simulation beginning with Nomoto's progenitor and using the Swesty-Lattimer equation of state at high densities.

12. illustrates the difference in average neutrino energy from simplified source terms of WB and the full equation for a range of degeneracy parameters $\eta$. 


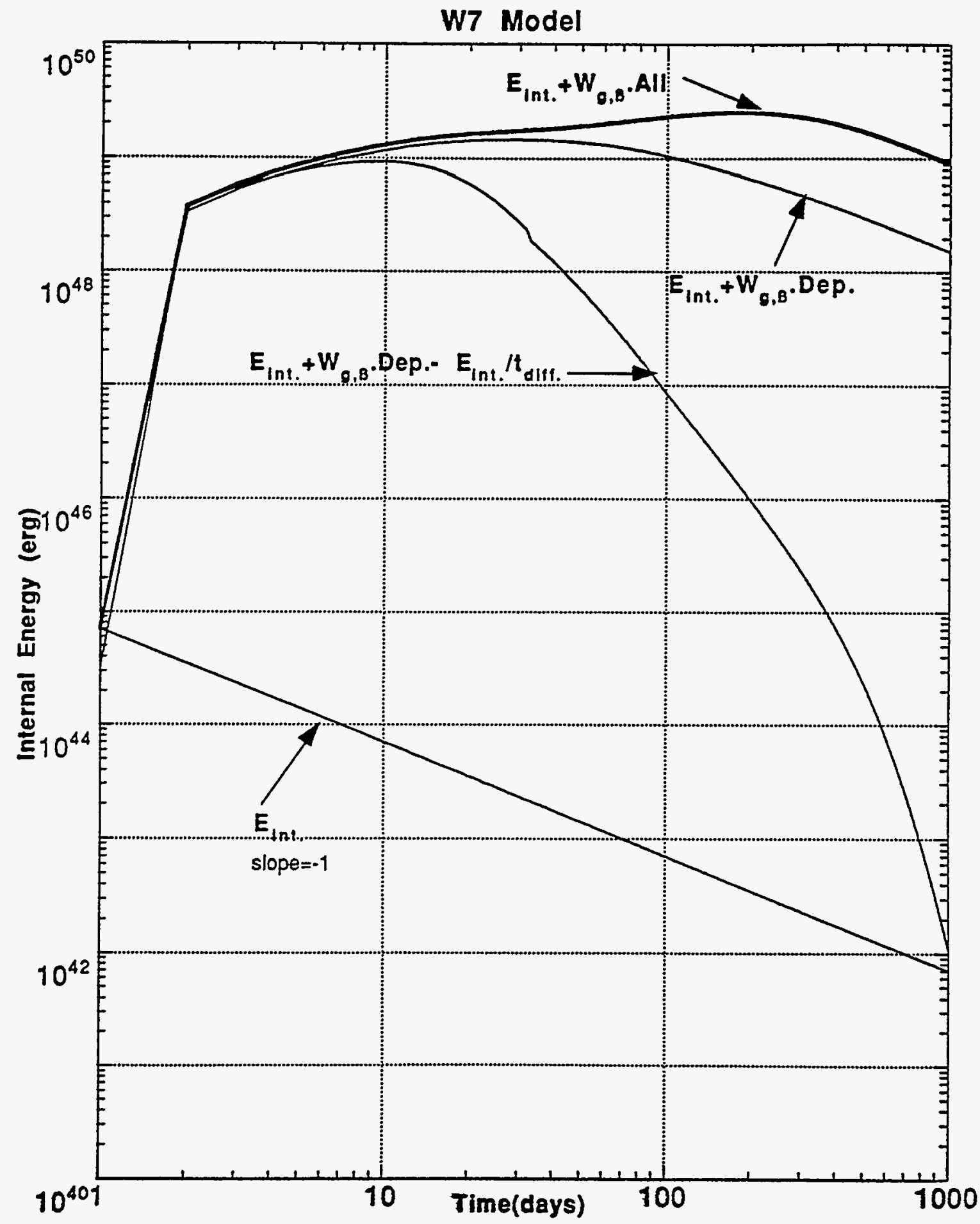


Opacity Function

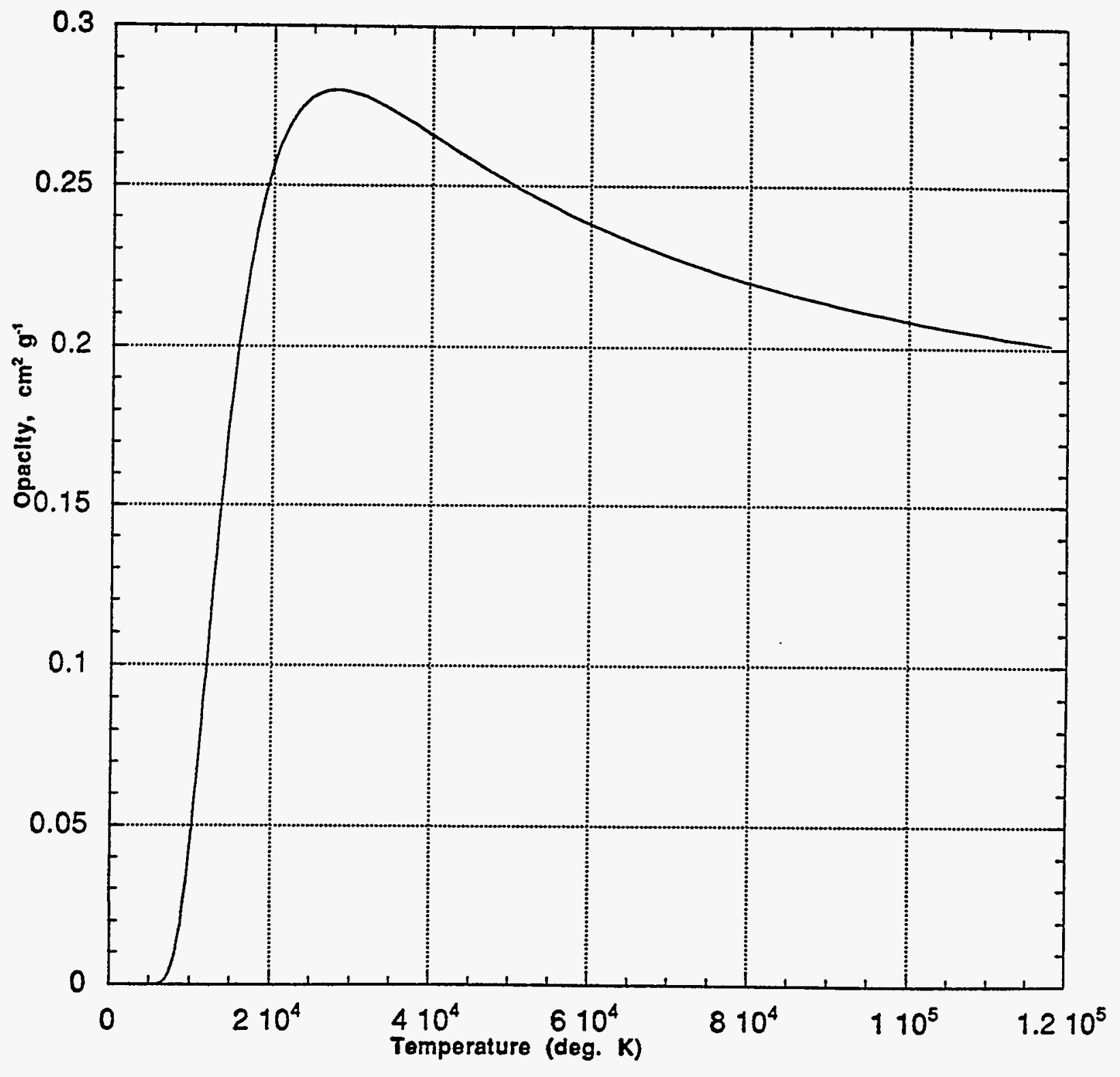




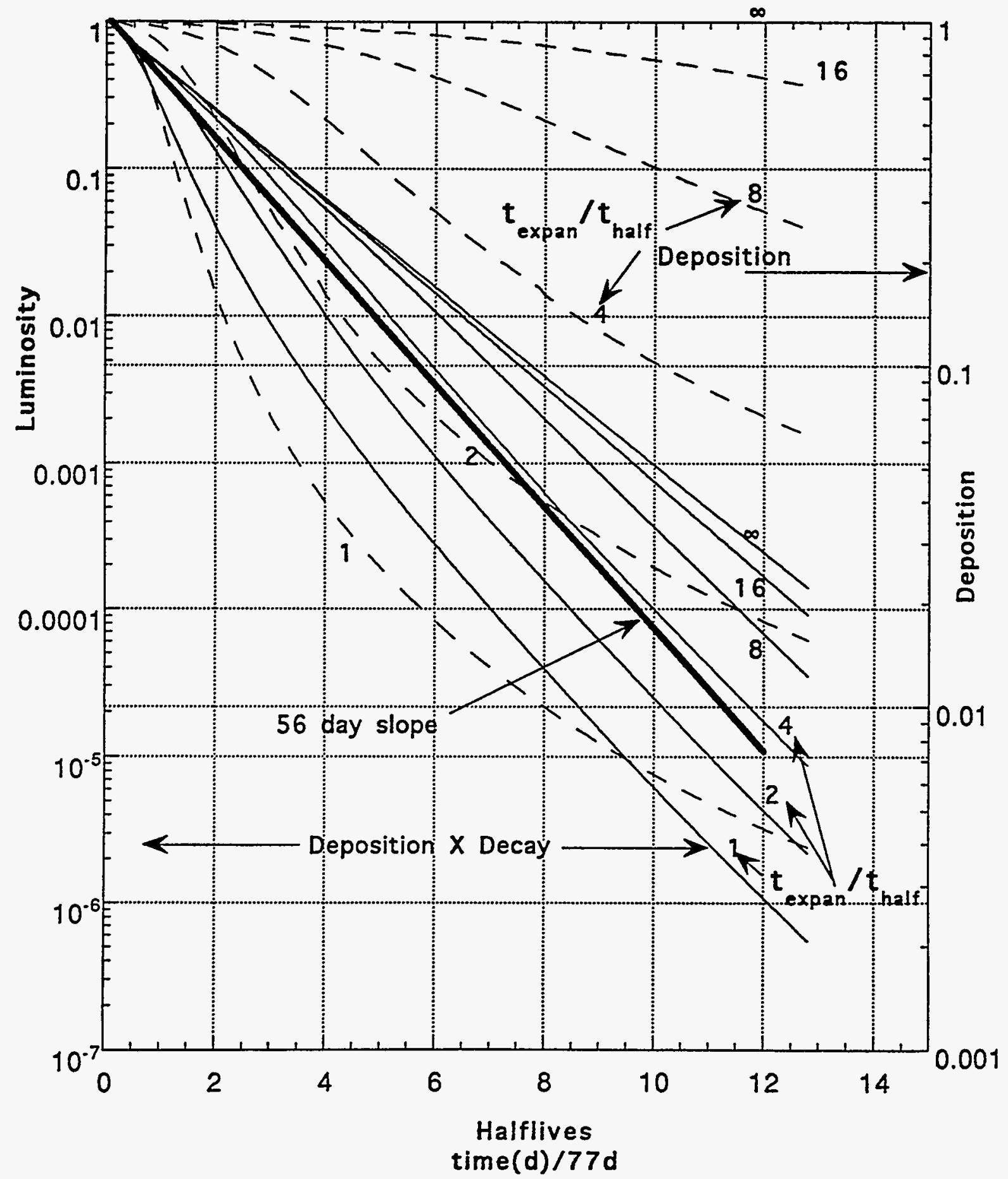




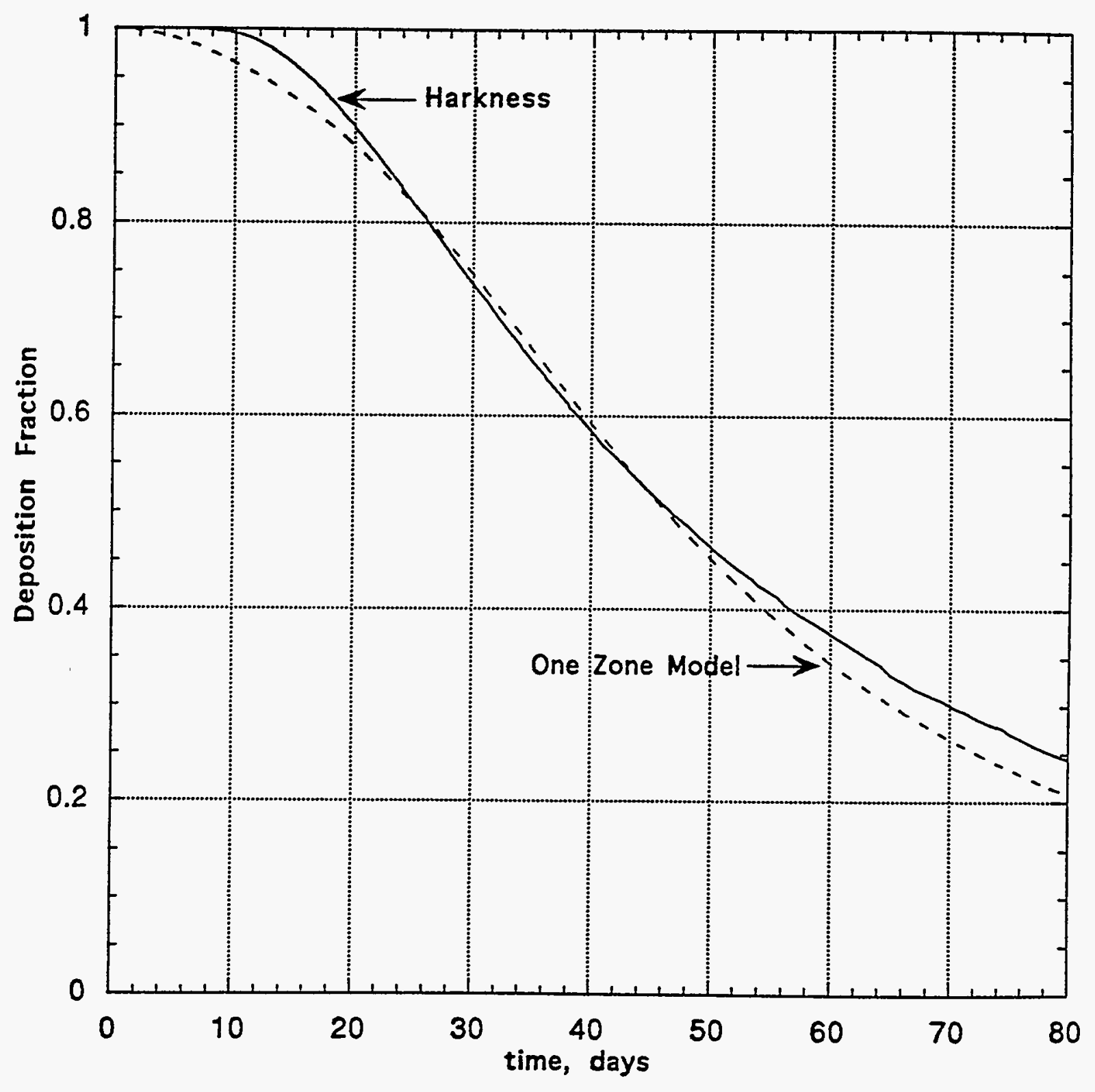




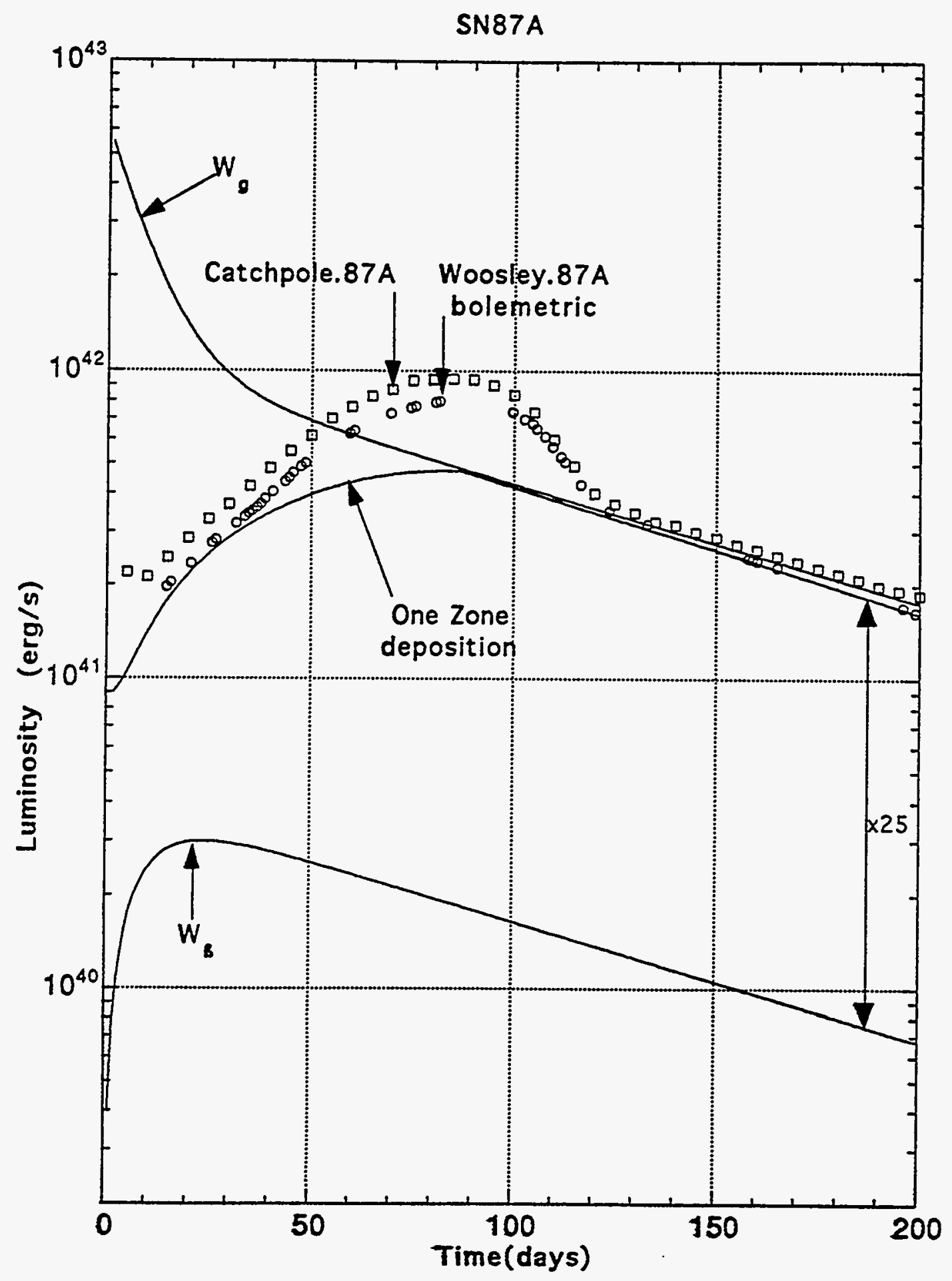




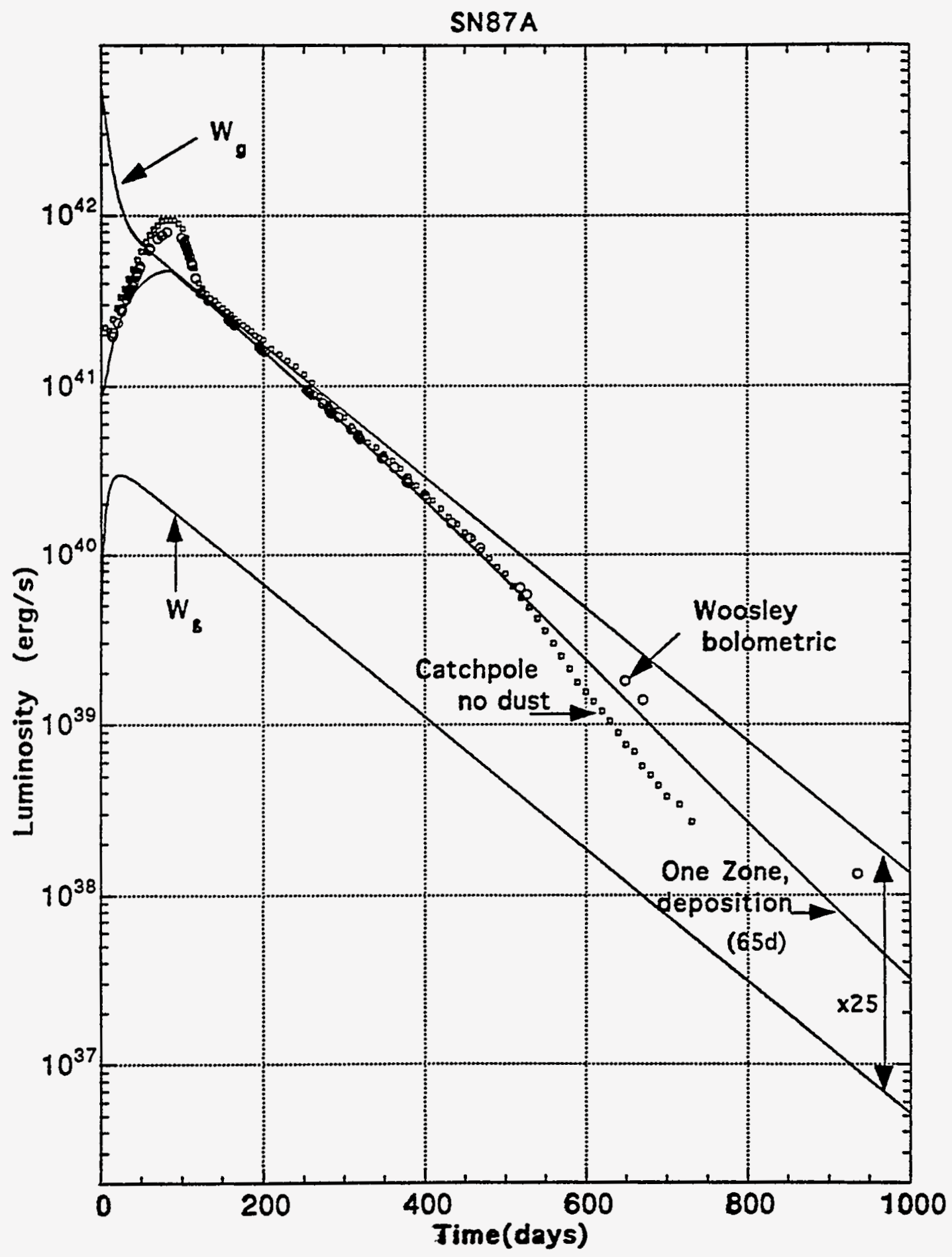




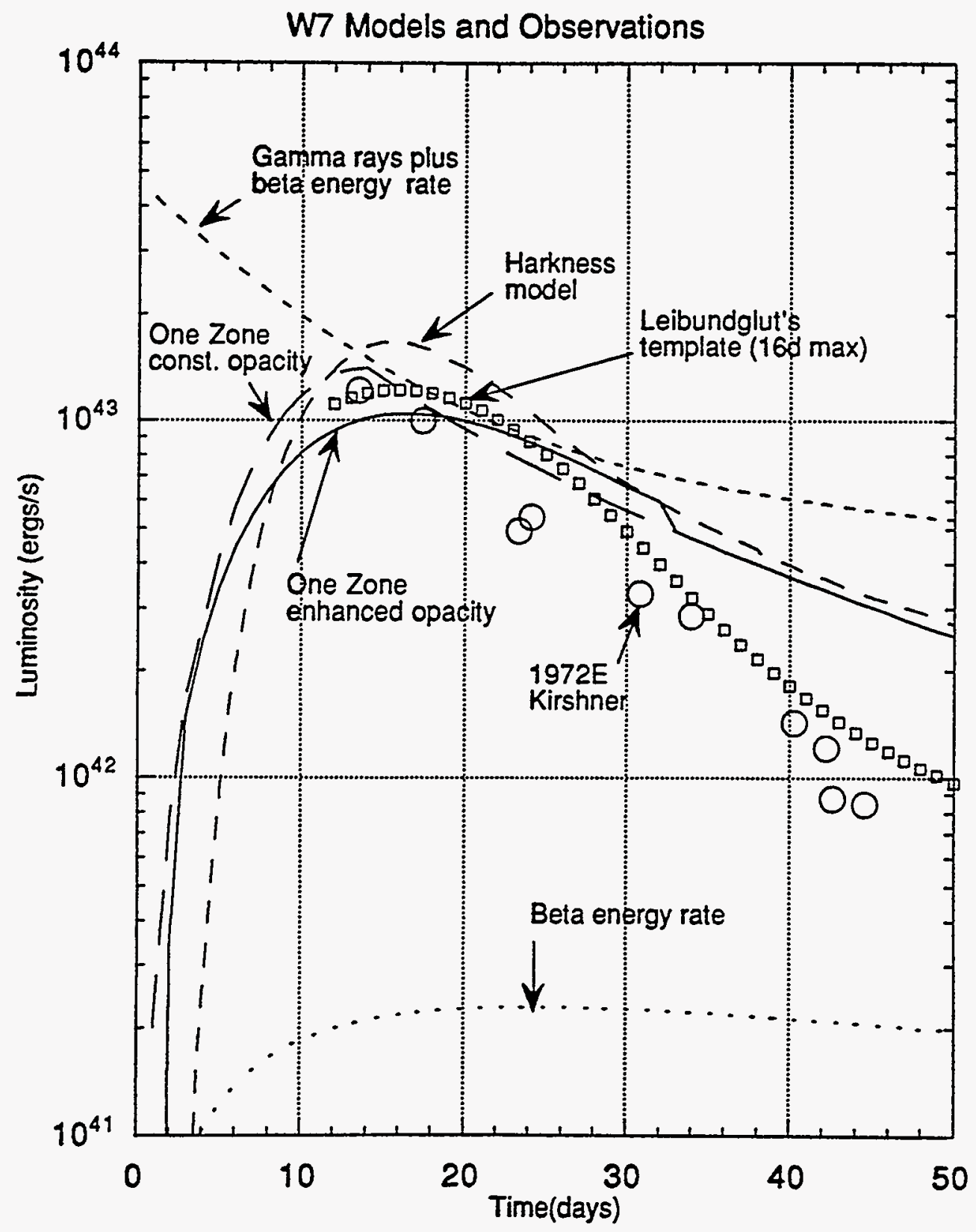




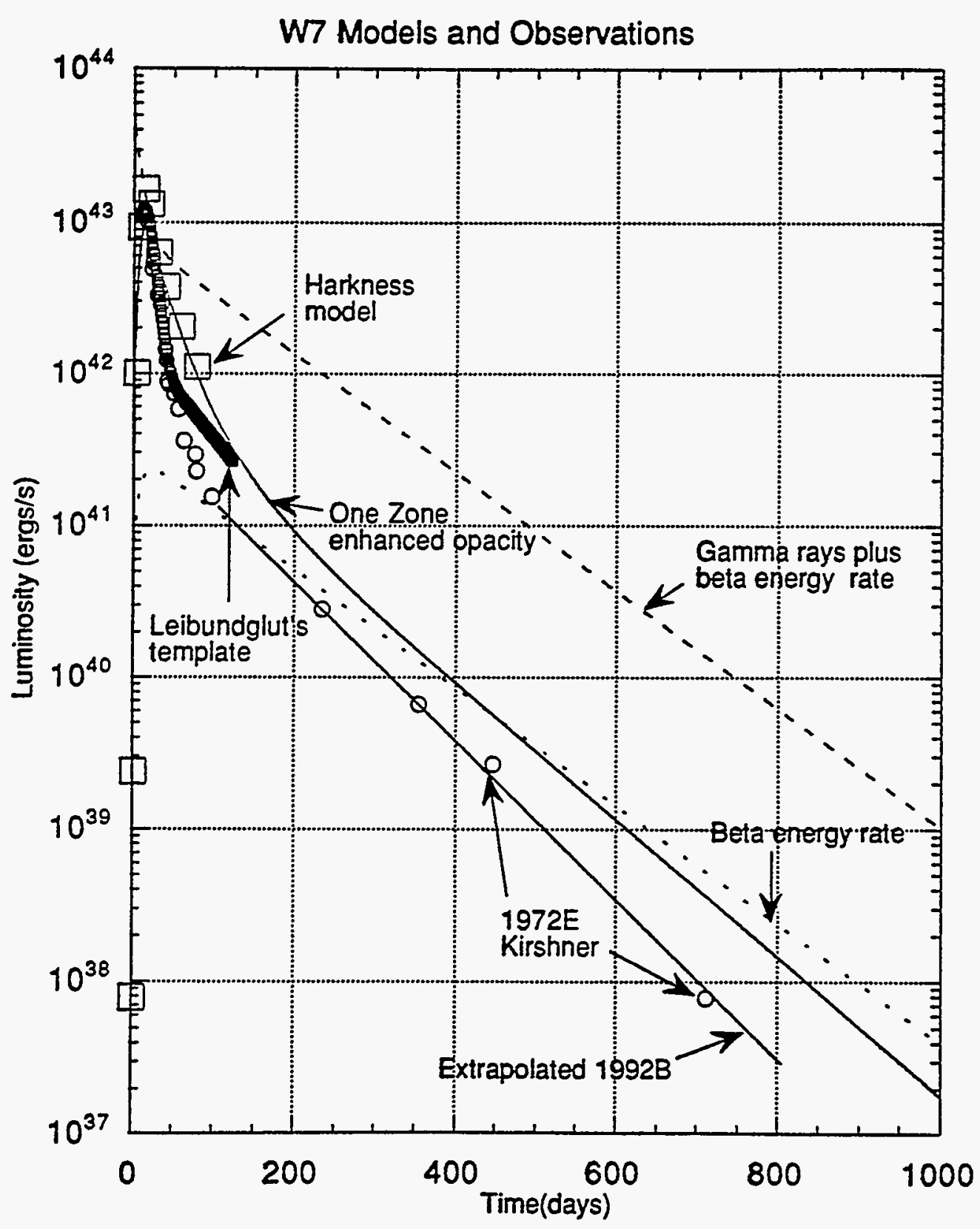




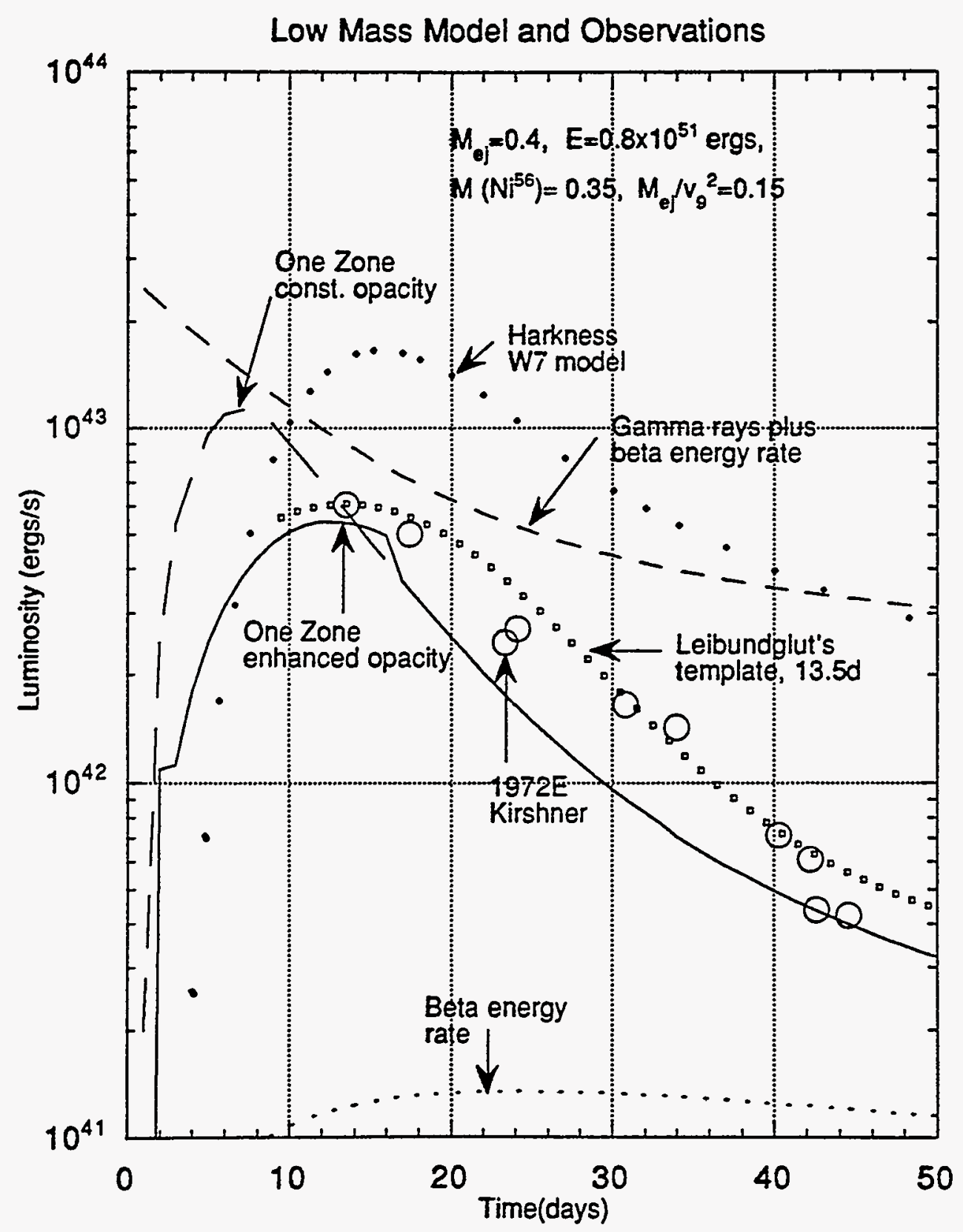




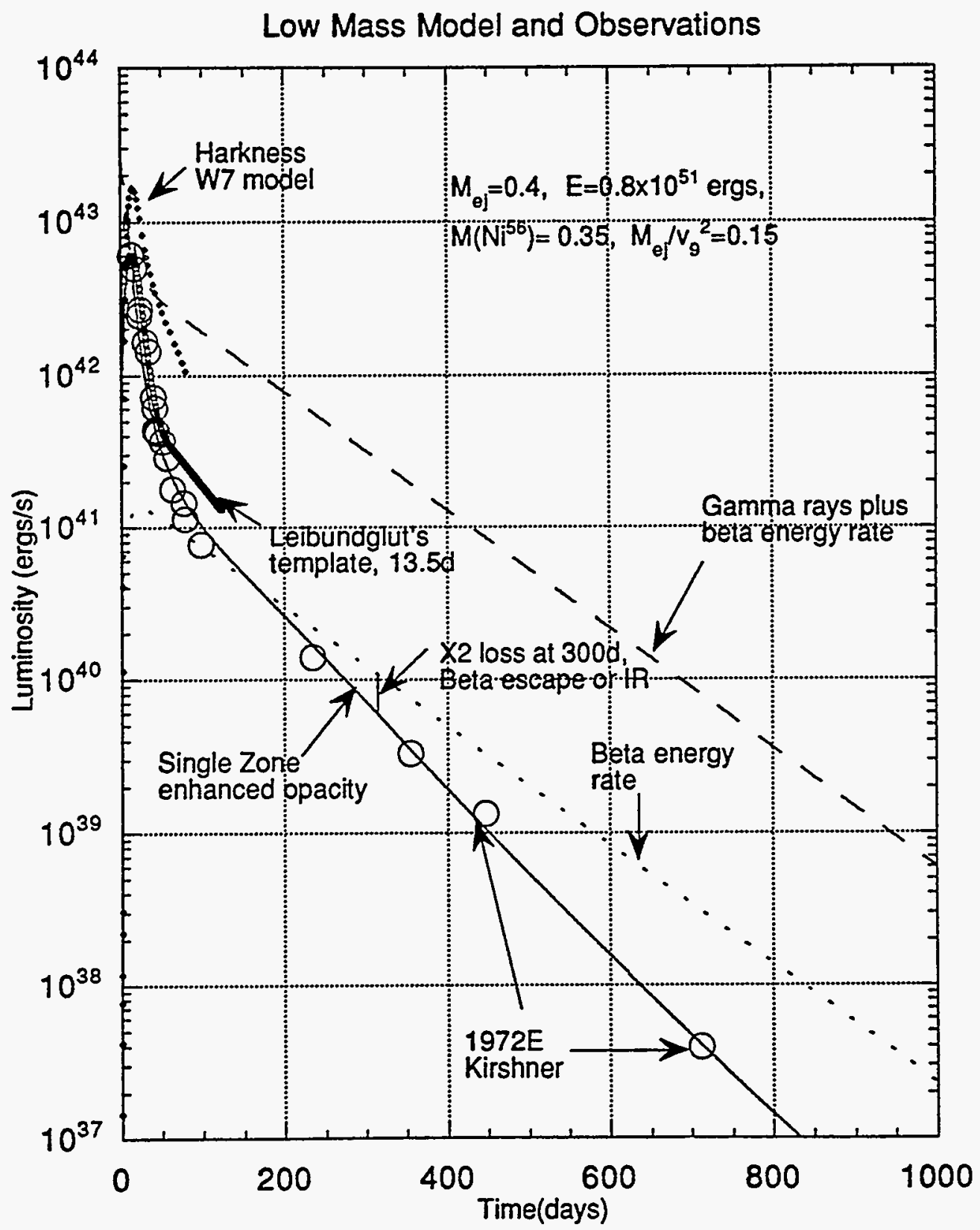

